\title{
GENERALIZED DPW METHOD AND AN APPLICATION TO ISOMETRIC IMMERSIONS OF SPACE FORMS
}

\author{
DAVID BRANDER AND JOSEF DORFMEISTER
}

\begin{abstract}
Let $G$ be a complex Lie group and $\Lambda G$ denote the group of maps from the unit circle $\mathbb{S}^{1}$ into $G$, of a suitable class. A differentiable map $F$ from a manifold $M$ into $\Lambda G$, is said to be of connection order $\left(\begin{array}{l}b \\ a\end{array}\right)$ if the Fourier expansion in the loop parameter $\lambda$ of the $\mathbb{S}^{1}$-family of Maurer-Cartan forms for $F$, namely $F_{\lambda}^{-1} \mathrm{~d} F_{\lambda}$, is of the form $\sum_{i=a}^{b} \alpha_{i} \lambda^{i}$. Most integrable systems in geometry are associated to such a map. Roughly speaking, the DPW method used a Birkhoff type splitting to reduce a harmonic map into a symmetric space, which can be represented by a certain order $\left({ }_{-1}^{1}\right)$ map, into a pair of simpler maps of order $\left(\begin{array}{l}-1 \\ -1\end{array}\right)$ and $\left(\begin{array}{l}1 \\ 1\end{array}\right)$ respectively. Conversely, one could construct such a harmonic map from any pair of $\left(\begin{array}{l}-1 \\ -1\end{array}\right)$ and $\left(\begin{array}{l}1 \\ 1\end{array}\right)$ maps. This allowed a Weierstrass type description of harmonic maps into symmetric spaces. We extend this method to show that, for a large class of loop groups, a connection order $\left(\begin{array}{l}b \\ a\end{array}\right)$ map, for $a<0<b$, splits uniquely into a pair of $\left(\begin{array}{l}-1 \\ a\end{array}\right)$ and $\left(\begin{array}{l}b \\ 1\end{array}\right)$ maps. As an application, we show that constant non-zero curvature submanifolds with flat normal bundle of a sphere or hyperbolic space split into pairs of flat submanifolds, reducing the problem (at least locally) to the flat case. To extend the DPW method sufficiently to handle this problem requires a more general Iwasawa type splitting of the loop group, which we prove always holds at least locally.
\end{abstract}

\section{INTRODUCTION}

Over the past two or three decades, various techniques based on the Birkhoff and Iwasawa loop group factorizations have been used successfully to study integrable systems in geometry. This paper may be viewed as an attempt to clarify generally the class of problems to which they apply, in addition to considering a new application.

1.1. Limited connection order maps into loop groups. Let $G$ be a complex Lie group, with Lie algebra $\mathfrak{g}$, and $\Lambda G$ the group of maps from the unit circle $\mathbb{S}^{1}$ into $G$, with a topology that makes $\Lambda G$ a Banach Lie group which has some additional properties needed for the purposes of this paper. For a matrix group $G$, the Wiener topology has all these properties, and includes all applications the authors are aware of.

Let $M$ be a smooth manifold and $F$ a smooth function from $M$ into $\Lambda G$. Denote the group of such maps by $\Lambda G(M)$. F is often regarded as a family of smooth maps $F_{\lambda}$ from $M$ into $G$, parameterized by $\lambda \in \mathbb{S}^{1}$. In this sense "the Maurer-Cartan

2000 Mathematics Subject Classification. Primary 37K10, 37K25, 53C42, 53B25; Secondary $53 \mathrm{C} 35$.

Research supported by DFG grant DO 776. 
form for $F$ " is the family $A_{\lambda}:=F_{\lambda}^{-1} \mathrm{~d} F_{\lambda}$ of Maurer-Cartan forms for $F_{\lambda}$. The integrability condition for $A_{\lambda}$ is the Maurer-Cartan equation

$$
\mathrm{d} A_{\lambda}+A_{\lambda} \wedge A_{\lambda}=0,
$$

and this is satisfied for all $\lambda$ in $\mathbb{S}^{1}$. Conversely, consider a family $A_{\lambda}$ of 1 -forms on $M$, with values in $\mathfrak{g}$, such that the map from $M$ to the Banach Lie algebra Lie $\Lambda G=: \Lambda \mathfrak{g}$, associating to $p \in M$ the $\mathbb{S}^{1}$-family $A_{\lambda}(p)$, is smooth and which satisfies, in addition, (11) for all $\lambda$. Then, on any simply connected domain in $M$, there exists a family $F_{\lambda} \in \Lambda G(M)$ whose Maurer-Cartan form is $A_{\lambda}$.

If we expand $A_{\lambda}$ as a Fourier series in $\lambda$,

$$
A_{\lambda}=\sum_{i} a_{i} \lambda^{i}
$$

where each $a_{i}$ is a $\mathfrak{g}$-valued 1 -form on $M$, then the equation (1) is equivalent to the system of equations

$$
\mathrm{d} a_{k}+\sum_{i+j=k} a_{i} \wedge a_{j}=0 .
$$

By assuming that $F$ is a map into a particular subgroup $\mathcal{H}$ of $\Lambda G$, for some particular group $G$, and that only a few $a_{i}$ are non-zero, it has been found that certain maps of interest in geometry - such as harmonic maps into symmetric spaces, 24, [30], 20], 37, [11, [10], isometric immersions of space forms into space forms [19], 18, 8], Hamiltonian stationary Lagrangian surfaces in $\mathbb{C}^{2}$ 23. - can be characterized by such an $F$, where the the special equations defining the particular type of map are precisely (2). A recent survey which catalogues most of the known examples is given by Terng 32 . More specific information concerning the construction of surface classes can be found in 13 .

We will say that an element $F \in \Lambda G(M)$ is of connection order $\left(\begin{array}{c}b \\ a\end{array}\right)$, where $a$ and $b$ are extended integers and $a \leq b$, if the Maurer-Cartan form of $F$ is a Laurent series of top and bottom degree $b$ and $a$ respectively,

$$
F^{-1} \mathrm{~d} F=\sum_{a}^{b} a_{i} \lambda^{i},
$$

and denote the subset of such maps by $\Lambda G(M)_{a}^{b}$. These are the basic objects of study in this article.

Let $\Lambda^{+} G$ and $\Lambda^{-} G$ denote the subgroups of $\Lambda G$ consisting of loops which extend to holomorphic maps into $G$ from the unit disc and its complement in the Riemann sphere respectively. Involutions of the loop group $\Lambda G$ can be divided into two basic types, those of the first and those of the second kind. These are essentially characterized by the property that they map $\Lambda^{ \pm} G$ to $\Lambda^{ \pm} G$ for the first kind, and $\Lambda^{ \pm} G$ to $\Lambda^{\mp} G$ for the second kind. An important subclass of limited connection order maps are the connection order $\left({ }_{-b}^{b}\right)$ maps with involution of the second kind $\tau$, or $\left({ }_{-b}^{b}\right)_{\tau}$ maps, where $b \geq 0$. These are maps into a subgroup $\Lambda G_{\tau}$, the fixed point set of an involution of the second kind $\tau$. In most applications that the authors are aware of, one seeks to construct maps into a real form $G \mathbf{R}$ of the complex Lie group $G$, and this leads to consideration of loops (actually maps from from $\mathbb{C}^{*}$ into $G$ ) which are real along either a line through the origin or along $\mathbb{S}^{1}$. The $\mathbb{S}^{1}$ reality condition is of the second kind, and so leads to type $\left({ }_{-b}^{b}\right)_{\tau}$ maps; a different example of a $\left({ }_{-b}^{b}\right)_{\tau}$ map will be discussed below, in Section 6 . 
For reasons which will become clear, we distinguish between two types of loop group problems: those with and those without an involution of the second kind.

1.2. Examples without an involution of the second kind. The $(b, j)$ 'th flow of the $G$-hierarchy 32 originating in the well known ZS-AKNS construction 38, [1], is associated to an order $\left(\begin{array}{l}j \\ 0\end{array}\right)$ map. The -1 -flow and the hyperbolic $U$-system [32] are $\left({ }_{-1}^{1}\right)$ maps. Some known examples of geometrical problems of such type are pseudospherical surfaces in $\mathbf{R}^{3}$ 34, 35, 36, and affine spheres 14, both of order $\left({ }_{-1}^{1}\right)$, and curved flats [18, which are of order $\left(\begin{array}{l}1 \\ 0\end{array}\right)$. Particular examples of curved flats are flat isometric immersions into a sphere or hyperbolic space [8] and isothermic surfaces [12].

1.3. Examples with an involution of the second kind. Harmonic maps into symmetric spaces are of connection order $\left({ }_{-1}^{1}\right)_{\tau}$, and, more generally, the $m$ 'th elliptic $(G, \tau)$ and $(G, \tau, \sigma)$ systems defined by Terng [32] are of order $(\underset{-m}{m})_{\tau}$. Some concrete examples are Hamiltonian stationary Lagrangian surfaces in $\mathbb{C}^{2}$, which are of order $\left({ }_{-2}^{2}\right)_{\tau}[23$, and isometric immersions with flat normal bundle of non-flat space forms into a sphere or hyperbolic space, which are of order $\left({ }_{-1}^{1}\right)_{\tau}$ [19] (see also Section 6 below).

1.4. The Birkhoff factorization theorem. The basis of the DPW method is the Birkhoff factorization theorem for $\Lambda G$ (Theorem 2.1), which states that: each element of $\Lambda G$ can be written in the form $g=g_{-} w g_{+}$, where $g_{ \pm} \in \Lambda^{ \pm} G$ and $w$ is in a specific set (which is in most of the cases of interest to us the Weyl group of the Lie algebra $\Lambda \mathfrak{g}$ ). Moreover, the left big cell, $\mathcal{L B} \Lambda G=\Lambda G^{-} \cdot \Lambda G^{+}$is an open and dense subset of $\Lambda G$. By definition, $\mathcal{L B} \Lambda G$ consists exactly of those elements of $g \in \Lambda G$, which can be written in the form $g=g_{-} g_{+}$, where $g_{ \pm} \in \Lambda G^{ \pm}$. We note that such a decomposition can be normalized in various ways. Usually we use $g_{+}(0)=I$ or $g_{-}(\infty)=I$ to make the decomposition unique. In this case, $g_{+}$and $g_{-}$are analytic functions of $g$. There is obviously an analogous statement with \pm interchanged. The corresponding right big cell will be denoted $\mathcal{R B} \Lambda G$. The intersection $\mathcal{B} \Lambda G=\mathcal{L B} \Lambda G \cap \mathcal{R B} \Lambda G$ will be of particular importance. It will be called the (central) big cell.

For certain purposes it will also turn out to be useful to consider groups more general than the loop groups discussed above. In particular, if such a group is a subgroup $\mathcal{H}$ of $\Lambda G$, we define subgroups $\mathcal{H}^{ \pm}=\mathcal{H} \cap \Lambda^{ \pm} G$.

We will call a subgroup $\mathcal{H}$ of $\Lambda G$ Birkhoff decomposable if the (central) big cell $\mathcal{B H}:=\left(\mathcal{H}^{+} \cdot \mathcal{H}^{-}\right) \cap\left(\mathcal{H}^{-} \cdot \mathcal{H}^{+}\right)$is open and dense in $\mathcal{H}$. Well known examples of Birkhoff decomposable groups are the $\sigma$-twisted subgroups $\Lambda G_{\sigma}$, which denotes a fixed point subgroup of $\Lambda G$, with respect to an involution of the first kind. But we will also present, for example in Section 2.2. Birkhoff decomposable groups different from those just mentioned.

In most cases, as considered in this paper, the loop groups which have arisen in integrable systems are either Birkhoff decomposable or a fixed point subgroup $\mathcal{H}_{\tau}$, where $\mathcal{H}$ is Birkhoff decomposable and $\tau$ is an involution of $\mathcal{H}$ of the second kind.

1.5. The generalized DPW method. The theme of this paper is to use Birkhoff decompositions to break down connection order $\left(\begin{array}{l}b \\ a\end{array}\right)$ maps (where $a<0<b$ ) into pairs of $\left(\begin{array}{l}-1 \\ a\end{array}\right)$ and $\left(\begin{array}{l}b \\ 1\end{array}\right)$ maps. This generalizes the DPW method [16], where the idea was applied to harmonic maps, an order $\left({ }_{-1}^{1}\right)_{\tau}$ example. In fact the pair of 
$\left(\begin{array}{l}1 \\ 1\end{array}\right)$ and $\left(\begin{array}{l}-1 \\ -1\end{array}\right)$ maps obtained in that example were related by the involution $\tau$, so the solution could be described by a single order $\left(\begin{array}{l}1 \\ 1\end{array}\right)$ map, whose Maurer-Cartan form turned out to be a meromorphic Lie algebra valued function of one complex variable.

We first prove (Propositions 3.1 and 3.2) that if $a<0<b$, and $\mathcal{H}$ is any Birkhoff decomposable subgroup, then connection order $\left(\begin{array}{l}b \\ a\end{array}\right)$ maps into $\mathcal{H}$ are in one to one correspondence with pairs of connection order $\left(\begin{array}{l}-1 \\ a\end{array}\right)$ and $\left(\begin{array}{l}b \\ 1\end{array}\right)$ maps. More accurately, the converse part, Proposition 3.2 allows one to construct an order $\left(\begin{array}{l}b \\ a\end{array}\right)$ map from a pair of $\left(\begin{array}{l}x \\ a\end{array}\right)$ and $\left(\begin{array}{l}b \\ y\end{array}\right)$ maps, where $x \geq a$ and $y \leq b$ are arbitrary extended integers. Propositions 3.1 and 3.2 are a straightforward generalization of similar results in 34, where they were proved for a loop group associated to pseudospherical surfaces in $\mathbf{R}^{3}$. In that case, the resulting pair of $\left(\begin{array}{c}-1 \\ -1\end{array}\right)$ and $\left(\begin{array}{l}1 \\ 1\end{array}\right)$ maps were each a Lie algebra valued function of one variable only, in asymptotic coordinates, which enabled a simplified "Weierstrass-type" characterization of pseudospherical surfaces. A similar outcome was obtained in [14] for affine spheres. Essentially the same idea had been used by Krichever in 27, before the introduction of the loop group point of view.

As noted above, many of the known applications are of order $\left({ }_{-b}^{b}\right)_{\tau}$, that is they map into a subgroup $\mathcal{H}_{\tau}$, where $\mathcal{H}$ is Birkhoff decomposable and where $\tau$ is an involution of $\mathcal{H}$ of the second kind. It is easy to see that $\mathcal{H}_{\tau}$ is not Birkhoff decomposable: this makes the converse part of the splitting tricky. We will show that the problem can be overcome when $\mathcal{H}$ is $\tau$-Iwasawa decomposable, that is, if a neighbourhood of the identity of $\mathcal{H}$ has a unique factorization

$$
F_{\tau} g_{+}
$$

where $F_{\tau}$ is an element of $\mathcal{H}_{\tau}$ and $g_{+}$is in $\mathcal{H}^{+}$. For such a situation, we prove (Proposition 3.4) that, in fact, there is a correspondence between connection order $\left(\begin{array}{c}b \\ -b\end{array}\right)_{\tau}$ maps and single order $\left(\begin{array}{l}b \\ 1\end{array}\right)$ maps.

It turns out (Theorem 2.11) that $\mathcal{H}$ is always $\tau$-Iwasawa decomposable, for any Birkhoff decomposable subgroup $\mathcal{H}$, and we can make more precise statements in the case that the subgroup of constant loops, $\mathcal{H}^{0}$, is compact.

We note here (see Remark 2.13), that, by considering the double loop group of [17, one can recast these results so that one only need consider involutions of the first kind.

In Section 5 we include a short discussion on how dressing defines a natural local action by $\mathcal{H}^{-}$on $\mathcal{H}(M)_{1}^{b}$, and similarly an action by $\mathcal{H}^{-} \times \mathcal{H}^{+}$on $\mathcal{H}(M)_{a}^{b}$.

In the last sections, we apply the splitting results to the cases of isometric immersions with flat normal bundle of space forms $M_{c} \rightarrow \widetilde{M}_{\tilde{c}}$, where $c$ and $\tilde{c}$ are the respective constant sectional curvatures. This problem had earlier been studied in the loop group framework, in [19], for non-flat immersions, and in [8] and [32] for the flat case.

We show that, given a choice of base point for the manifold, the DPW splitting produces (at least at a local level) a 1-1-relation between all non-flat immersions and all flat immersions, in accordance with Table 1.

As another application of the generalized DPW method described here, the technique is used, in the article [6], to relate isometric immersions of space forms to pluriharmonic maps, and that particular setup is generalized further to constant curvature Lagrangian submanifolds of space forms in [7]. 
TABLE 1. Local correspondence between non-flat and flat immersions, $M_{c} \rightarrow \widetilde{M}_{\tilde{c}}$

\begin{tabular}{|c|c||c||c|c|}
\hline$c \in(-\infty, 0)$ & $\tilde{c}=1$ & $\leftrightarrow$ & $c=0$ & $\tilde{c}=1$ \\
\hline$c \in(0,1)$ & $\tilde{c}=1$ & $\leftrightarrow$ & $c=0$ & $\tilde{c}=1$ \\
\hline$c \in(1, \infty)$ & $\tilde{c}=1$ & $\leftrightarrow$ & $c=0$ & $\tilde{c}=-1$ \\
\hline$c \in(-\infty,-1)$ & $\tilde{c}=-1$ & $\leftrightarrow$ & $c=0$ & $\tilde{c}=1$ \\
\hline$c \in(-1,0)$ & $\tilde{c}=-1$ & $\leftrightarrow$ & $c=0$ & $\tilde{c}=-1$ \\
\hline$c \in(0, \infty)$ & $\tilde{c}=-1$ & $\leftrightarrow$ & $c=0$ & $\tilde{c}=-1$ \\
\hline
\end{tabular}

\section{BIRKHOFF AND $\tau$-IWASAWA DECOMPOSITIONS FOR SUBgRoups OF $\Lambda G$}

In the next few sections, we discuss some general theory of (loop) group decompositions and their applications to connection order $\left(\begin{array}{l}b \\ a\end{array}\right)$ maps. Throughout this discussion, $\Lambda G$ will generally denote a loop group, and $\mathcal{H}$ some Birkhoff decomposable subgroup of $\Lambda G$.

2.1. Definitions and terminology. For convenience, we gather together here some definitions.

2.1.1. The loop group. All groups of interest to us can be realized as matrix groups, so we proceed as follows: let $G$ be a complex semisimple matrix Lie group, with some matrix norm $\|\cdot\|$, satisfying $\|A B\| \leq\|A\|\|B\|$ and $\|I\|=1$, and with Lie algebra $\mathfrak{g}$. Define the loop group

$$
\Lambda G:=\left\{g: \mathbb{S}^{1} \rightarrow G \mid g=\sum_{i=-\infty}^{\infty} g_{i} \lambda^{i}, \quad \sum\left\|g_{i}\right\|<\infty\right\} .
$$

Equipped with the topology induced by the norm $\|g\|:=\sum\left\|g_{i}\right\|, \Lambda G$ is a Banach Lie group whose Lie algebra, $\Lambda \mathfrak{g}$, consists of power series in $\lambda$ with coefficients in the Lie algebra of $G$, and the corresponding convergence condition. For more details see [2] or [31].

2.1.2. Subgroups. All subgroups of $\Lambda G$ are assumed to be Banach Lie subgroups in the sense of [5]. In particular, subgroups are closed submanifolds and carry the induced topology. Let $\widehat{\mathbf{C}}$ be the extended plane, $\mathbf{D}_{+}$the open unit disc, and $\mathbf{D}_{-}$ the complement of its closure in $\widehat{\mathbf{C}}$. For any group $G$ as above, define the following subgroups of $\Lambda G$ :

$$
\Lambda^{ \pm} G:=\left\{g \in \Lambda G \mid g \text { has a holomorphic extension } \hat{g}: \mathbb{D}_{ \pm} \rightarrow G\right\} .
$$

These have the subgroups $\Lambda_{1}^{+} G$ and $\Lambda_{1}^{-} G$ whose elements have the respective normalizations $g(0)=I$ and $g(\infty)=I$.

For a subgroup $\mathcal{H}$ of $\Lambda G$, we define $\mathcal{H}^{ \pm}$and $\mathcal{H}_{1}^{ \pm}$to be the intersection of $\mathcal{H}$ with the notationally corresponding subgroups of $\Lambda G$. In all concrete examples we consider, all these groups will be Banach lie groups and subgroups respectively.

If $\sigma$ and $\rho$ are automorphisms of $\mathcal{H}$, then $\mathcal{H}_{\sigma \rho}$ denotes the subgroup of $\mathcal{H}$ consisting of elements fixed by both automorphisms, and similar notation is used for any number of automorphisms. In particular, $\Lambda G_{\sigma}$ means $(\Lambda G)_{\sigma}$. 
2.2. Birkhoff factorization. The Birkhoff factorization theorem, as stated here, is proved, for the case of $\mathcal{C}^{\infty}$ loops in 31 for $G L(n, \mathbb{C})$ as well as for any complex semisimple Lie group. In [2], a similar result is shown to hold, working with the Wiener topology, for the complexification of any connected Lie group which admits a faithful finite dimensional continuous representation.

Theorem 2.1. (1) Any loop $g \in \Lambda G$ has a right Birkhoff factorization

$$
g=g_{+} D g_{-},
$$

where $g_{+} \in \Lambda^{+} G, g_{-} \in \Lambda^{-} G$ and $D$ is a homomorphism from $\mathbb{S}^{1}$ into a maximal torus of $G$. Similarly a left Birkhoff factorization,

$$
g=g_{-} D g_{+}
$$

exists, where $g_{-} \in \Lambda^{-} G$ and $g_{+} \in \Lambda^{+} G$.

(2) The left big cell, $\mathcal{L B} \Lambda G$, defined to be the subset on which the left factorizations have $D=I$ as the center term, is open and dense in $\Lambda G$. In fact it is given as the complement of the zero set of a non-constant holomorphic function $f_{L}: \Lambda G \rightarrow \mathbb{C}$. The right big cell has the analogous definition and properties.

(3) The maps $\Lambda_{1}^{+} G \times \Lambda^{-} G \rightarrow \mathcal{R B} \Lambda G$, and $\Lambda_{1}^{-} G \times \Lambda^{+} G \rightarrow \mathcal{L B} \Lambda G$ are analytic diffeomorphisms as are the maps $\Lambda^{+} G \times \Lambda_{1}^{-} G \rightarrow \mathcal{R B} \Lambda G$ and $\Lambda^{-} G \times \Lambda_{1}^{+} G \rightarrow$ $\mathcal{L B} \Lambda G$. In particular, on the big cells the factorization is unique, if one requires $g_{+} \in \Lambda_{1}^{+} G$ or $g_{-} \in \Lambda_{1}^{-} G$.

The intersection, $\mathcal{B} \Lambda G:=\mathcal{L} \mathcal{B} \Lambda G \cap \mathcal{R B} \Lambda G$, we will call the (central) big cell.

More generally, a Banach Lie group $\mathcal{H}$ together with Banach Lie subgroups subgroups $\mathcal{H}_{1}^{ \pm}$and $\mathcal{H}^{\circ}$ will be called Birkhoff decomposable, if $\mathcal{R B H}:=\mathcal{H}_{1}^{+} \cdot \mathcal{H}^{\circ} \cdot \mathcal{H}_{1}^{-}$ and $\mathcal{L B H}:=\mathcal{H}_{1}^{-} \cdot \mathcal{H}^{\circ} \cdot \mathcal{H}_{1}^{+}$are open and dense in $\mathcal{H}$ and the maps $\mathcal{H}_{1}^{+} \times \mathcal{H}^{\circ} \times \mathcal{H}_{1}^{-} \rightarrow$ $\mathcal{R B H}$ and $\mathcal{H}_{1}^{-} \times \mathcal{H}^{\circ} \times \mathcal{H}_{1}^{+} \rightarrow \mathcal{R B H}$ are analytic diffeomorphisms. In this case every element $h \in \mathcal{R B H}$ has a unique decomposition $h=h_{1}^{+} \cdot h^{\circ} \cdot h_{1}^{-}$with $h_{1}^{ \pm} \in \mathcal{H}_{1}^{ \pm}$and $h^{\circ} \in \mathcal{H}^{\circ}$. The analogous statement for elements in $h \in \mathcal{L B H}$ also holds. Setting $\mathcal{H}^{+}=\mathcal{H}_{1}^{+} \cdot \mathcal{H}^{\circ}$ and $\mathcal{H}^{-}=\mathcal{H}_{1}^{-} \cdot \mathcal{H}^{\circ}$ we obtain the properties listed in the last part of Theorem 2.1.

All examples of Birkhoff decomposable subgroups considered in this paper will be related in one way or another to subgroups of loop groups.

Let $\mathcal{H} \subset \Lambda G$ be a Banach Lie subgroup.

Definition 2.2. We will call $\mathcal{H}$ a Birkhoff decomposable subgroup of $\Lambda G$ if $\mathcal{H}_{1}^{+}:=$ $\mathcal{H} \cap \Lambda G_{1}^{+}, \mathcal{H}_{1}^{-}:=\mathcal{H} \cap \Lambda G_{1}^{-}$and $\mathcal{H}^{\circ}:=\mathcal{H} \cap \Lambda^{+} G \cap \Lambda^{-} G$ are close Banach Lie subgroups and make $\mathcal{H}$ into a Birkhoff decomposable group.

Example 1 The groups $\Lambda G$ are Birkhoff decomposable groups relative to $\Lambda G_{1}^{+}$, $\Lambda G_{1}^{-}$and $\Lambda G^{\circ}=\Lambda G^{+} \cap \Lambda G^{-}=G$.

Example 2 Let $\left(\mathcal{H}, \mathcal{H}_{1}^{+}, \mathcal{H}^{\circ}, \mathcal{H}_{1}^{-}\right)$and $\left(\mathcal{K}, \mathcal{K}_{1}^{+}, \mathcal{K}^{\circ}, \mathcal{K}_{1}^{-}\right)$be Birkhoff decomposable groups. Then the product group, together with the obvious subgroups is also a Birkhoff decomposable group.

Example 3 Let $\left(\mathcal{H}, \mathcal{H}_{1}^{+}, \mathcal{H}^{\circ}, \mathcal{H}_{1}^{-}\right)$be a Birkhoff decomposable subgroup of some loop group $\Lambda G$.

Theorem 2.3. Set

$$
\begin{aligned}
\mathcal{K}:=\mathcal{H} \times \mathcal{H}, & \mathcal{K}^{\circ}=\mathcal{H}^{\circ} \times \mathcal{H}^{\circ}, \\
\mathcal{K}_{1}^{+}=\mathcal{H}_{1}^{+} \times \mathcal{H}_{1}^{-} & \mathcal{K}_{1}^{-}=\left\{(x, x) ; x \in \mathcal{H},\left.x\right|_{\lambda=1}=I\right\} .
\end{aligned}
$$


Then $\mathcal{K}$ with the corresponding subgroups is a Birkhoff decomposable group.

Proof. We note first that $\mathcal{K}$ is a Banach Lie group and the remaining three groups are Banach Lie subgroups of $\mathcal{K}$. For $\mathcal{K}_{1}^{-}$one uses the fact that for loop groups with the Wiener topology, the evaluation map is an analytic homomorphism of Lie groups, such that its kernel is a Banach Lie subgroup (actually a normal subgroup, of course). The decisive object now is the map: $\Psi: \mathcal{K}_{1}^{-} \times \mathcal{K}^{\circ} \times \mathcal{K}_{1}^{+} \rightarrow \mathcal{K}$ given by $\left((h, h),(a, b),\left(g_{+}, g_{-}\right)\right) \mapsto\left(h a g_{+}, h b g_{-}\right)$. It is straightforward to prove that it is analytic and injective. Considering the inverse map we start from $(p, q)=\left(h a g_{+}, h b g_{-}\right)$. Then $p^{-1} q=g_{+}^{-1} a^{-1} b g_{-}$. Therefore, using that $\mathcal{H}$ is a Birkhoff decomposable group, $g_{+}$and $g_{-}$are analytic in $p$ and $q$ as well as $a^{-1} b$. But then the equation $p g_{+}^{-1}=h a$ shows, by evaluating at $\lambda=1$, that $a \in G$ is analytic in $p$ and $q$. Similarly one sees that $b$ is analytic in $p$ and $q$. Thus, finally, also $h$ is analytic in $p$ and $q$.

It remains to check that the image $\operatorname{Im} \Psi$ of $\Psi$ is open and dense in $\mathcal{K}$. This can be verified by observing that $(p, q)$ lies in $\operatorname{Im} \Psi$ if and only if we can write $p=h f_{+}$and $q=h f_{-}$, for some $h \in \mathcal{H}$ and $f_{ \pm} \in \mathcal{H}^{ \pm}$. This is equivalent to solving $p f_{+}^{-1}=q f_{-}^{-1}$, or $q^{-1} p=f_{-}^{-1} f_{+}$, which has a solution if and only if $q^{-1} p \in \mathcal{L B H}$. Therefore, the image of $\Psi$ is $\phi^{-1}(\mathcal{L B H})$, where $\phi(p, q):=q^{-1} p$ is a real analytic map, and $(p, q) \in \operatorname{Im} \Psi$ if and only if $f_{L} \circ \phi(p, q) \neq 0$, where $f_{L}$ is the holomorphic function given in Theorem 2.1. But $f_{L} \circ \phi$ is real analytic, and so the complement of its zero set is either empty or open and dense. It cannot be empty, as it contains the identity.

We will give more examples of Birkhoff decomposable groups in the following sections.

2.3. Automorphisms and Birkhoff decomposable subgroups. Let $\sigma: \Lambda G \rightarrow$ $\Lambda G$ be a real analytic automorphism, with fixed point subgroup $\Lambda G_{\sigma}$. Clearly, we can Birkhoff factorize elements in $\Lambda G_{\sigma}$ with factors in the larger group $\Lambda G$. We would like the factors to be in $\Lambda G_{\sigma}$ themselves, more strongly even, the subgroup to be Birkhoff decomposable. More generally, if $\mathcal{H}$ is any Birkhoff decomposable subgroup we ask the same question for fixed point subgroups $\mathcal{H}_{\sigma}$ of $\mathcal{H}$.

Finite automorphisms of $\Lambda G$ have been classified [28], 25], 3]. See also [22]. After passing to an isomorphic loop group, one can assume, for a complex linear automorphism $\phi$, of finite order $n$, that it can be expressed as

$$
(\phi x)(\lambda)=\phi_{0}\left(x\left(e^{\frac{2 \pi i}{n}} \lambda^{j}\right)\right)
$$

where $\phi_{0}$ is an automorphism of $G$ and $j= \pm 1$. If $j$ is positive, the automorphism is said to be of the first kind. If negative, of the second kind. In the latter case one can even assume that the factor $e^{\frac{2 \pi i}{n}}$ is not present. However, for simplicity of presentation we will not use this possibility in general.

If the automorphism $\rho$, of finite order $n$, is complex anti-linear, then one can assume it is of the form

$$
(\rho x)(\lambda):=\rho_{0}\left(x\left((-1)^{i}(\bar{\lambda})^{j}\right)\right), \quad i, j \in\{1,-1\},
$$

for some anti-linear involution $\rho_{0}$ of $G$. These are also said to be of the first and second kind if $j$ is positive or negative respectively. As above, for involutions of the second kind the factor $(-1)$ can be removed, but it is convenient not to do this. 
Remark 2.4. In fact there are applications where one is also interested in involutions which are not in standard form, such as the $n \times n \mathrm{KdV}$ reality condition used in 33. In this article, however, all automorphisms are assumed to be of the forms given by (5) and (6). We note that all the automorphisms of finite order used in this paper are analytic and their fixed point subgroups are Banach Lie subgroups.

A real form of $\Lambda G$ is defined to be the fixed point set of an anti-linear involution.

At the level of Lie algebras, one also has expressions of the form (5) and (6), replacing $\phi$ and $\rho$ with the corresponding Lie algebra automorphisms.

Note that an automorphism of the first kind takes $\Lambda^{ \pm} G \rightarrow \Lambda^{ \pm} G$, while one of the second kind takes $\Lambda^{ \pm} G \rightarrow \Lambda^{\mp} G$.

Proposition 2.5. Let $\mathcal{H}$ be a Birkhoff decomposable subgroup of $\Lambda G$, and suppose $\phi$ is a finite order automorphism of $\Lambda G$ which restricts to an automorphism of $\mathcal{H}$. Then:

(1) If $\phi$ is of the first kind, then $\mathcal{H}_{\phi}$ is Birkhoff decomposable.

(2) If $\phi$ is of the second kind, and $\mathcal{H}_{\phi}$ does not consist entirely of constant loops, then $\mathcal{H}_{\phi}$ is not Birkhoff decomposable.

Proof. (1) We give the proof for the right factorization here, the left being analogous. Suppose $x \in \mathcal{R} \mathcal{B H} \cap \mathcal{H}_{\phi}$. Since $x$ is fixed by $\phi$, we have $x=\phi x$. Since $x \in \mathcal{R B H}, x$ has a unique decomposition $x=x_{+} x^{\circ} x_{-}$with $x_{ \pm} \in \mathcal{H}_{1}^{ \pm}$ and $x^{\circ} \in \mathcal{H}^{\circ}$. First we show that these factors are already contained in $\mathcal{H}_{\phi}$ and that the required density and analyticity properties hold. First we note

$$
x_{+} x^{\circ} x_{-}=\phi x_{+} \phi x^{\circ} \phi x_{-} .
$$

Now $\phi$ takes $\mathcal{H}^{ \pm} \rightarrow \mathcal{H}^{ \pm}$and $\mathcal{H}^{\circ}$ to $\mathcal{H}^{\circ}$. As a consequence of this and the uniqueness of the factorization (7), $\phi$ fixes each of the three factors, so $x_{ \pm} \in \mathcal{H}_{\phi}^{ \pm}$and $x^{\circ} \in \mathcal{H}_{\phi}^{\circ}$.

Next we consider the map $\Sigma: \mathcal{H}_{\phi}^{+} \times \mathcal{H}_{\phi}^{\circ} \times \mathcal{H}_{\phi}^{-} \rightarrow \mathcal{R} \mathcal{B} \mathcal{H}_{\phi}$. We note that our argument above shows $\mathcal{R B H}_{\phi}=\mathcal{H}_{\phi} \cap \mathcal{R B} \Lambda G$. Since $\mathcal{H}_{\phi}$ carries the induced topology, $\mathcal{R B H}_{\phi}$ is open in $\mathcal{H}_{\phi}$. Moreover, since the map $\Sigma$ is the restriction of the corresponding map for $\Lambda G$ and since $\mathcal{H}_{\phi}$ is a Banach submanifold, it follows that $\Sigma$ is an analytic diffeomorphism. Finally, to prove that the image of the map under investigation is dense in $\mathcal{H}_{\phi}$, we use the fact that, according to Theorem 2.1, $\mathcal{R B} \Lambda G$ is given as the complement of the zero set of a holomorphic function, $f_{R}$, on $\Lambda G$. Hence $\mathcal{R} \mathcal{B H}_{\phi}$ is the complement of the zero set of $\left.g\right|_{\mathcal{H}_{\phi}}$, and this function is real analytic. Therefore, $\mathcal{R B H}_{\phi}$ is either empty or dense, and must be the latter, as it contains the identity.

(2) If $x$ is a non-constant loop in $\mathcal{H}_{\phi}$, then, again, we have the equation (77). Either $x_{+}$or $x_{-}$is a non-constant loop. Since $\phi$ is of the second kind, and takes $\mathcal{H}_{1}^{ \pm}$to $\mathcal{H}_{1}^{\mp}$, it is impossible that such a non-constant factor be fixed by $\phi$. That is, the factors cannot both be in $\mathcal{H}_{\phi}$.

Remark 2.6. Many of the subgroups explicitly referred to in this article (such as the $\sigma$-twisted subgroup $\Lambda G_{\sigma}$, where $\sigma$ is a complex linear involution of the first kind, induced from an inner involution of $G$ ) are in fact isomorphic to $\Lambda G$. However, it is 
important to note that the Birkhoff and Iwasawa decompositions we prove for these are not the same decompositions which would be obtained via this isomorphism and the standard decompositions for $\Lambda G$. For example, with $\Lambda G_{\sigma}$, the three relevant subgroups, namely the constant subgroup and $\Lambda^{ \pm} G_{\sigma}$, do not correspond exactly to the corresponding subgroups $G$ and $\Lambda^{ \pm} G$ under the isomorphism.

2.4. $\tau$-Iwasawa factorization. We saw that fixed point subgroups of automorphisms of the second kind are not Birkhoff decomposable. What we will later use in this situation, for the case that $\tau$ is of order 2, is a more general version of the Iwasawa factorization, which states that if $U$ is a compact real form of $G$, then any element of $\Lambda G$ can be written as a product $u g_{+}$, where $u \in \Lambda U$ and $g_{+} \in \Lambda^{+} G$.

It turns out that the discussion of (generalized) Iwasawa decomposable groups is much more complicated than the case of Birkhoff decomposable groups. The reason for this will become clear, when we discuss examples below.

To keep the presentation as simple and short as possible we start from a very basic definition:

Definition 2.7. Let $\mathcal{H}$ be some Banach Lie group, $\tau$ some real analytic involution and $\mathcal{H}_{+}$some Banach Lie subgroup. Then $\mathcal{H}$ together with the Banach Lie subgroups $\mathcal{H}_{\tau}$ and $\mathcal{H}_{+}$is called $\tau$-Iwasawa decomposable if $\mathcal{H}_{\tau} \cdot \mathcal{H}_{+}$is open in $\mathcal{H}$.

This definition even permits $\tau=i d$ and $\mathcal{H}_{+}=\mathcal{H}$. However, we will apply this notion below exclusively in situations where it is non-trivial.

Remark 2.8. 1. As in the case of Birkhoff decomposable groups one obtains a representation

$$
\mathcal{H}=\bigcup_{\iota \in \mathcal{J}} \mathcal{H}_{\tau} \cdot \iota \cdot \mathcal{H}_{+}
$$

where $\mathcal{J}$ is a set of representatives for the orbits of the group action by $\mathcal{H}_{\tau} \times \mathcal{H}_{+}$ on $\mathcal{H}$ given by $\left(h_{\tau}, h_{+}\right) \cdot h=h_{\tau} \cdot h h_{+}^{-1}$.

2. Kellersch [26], has investigated extensively Iwasawa decomposability in finite dimensional groups as well as in loop groups. He has given many examples illustrating what can happen.

3. If $\mathcal{H}$ is a Birkhoff decomposable subgroup of some loop group $\Lambda G$ and $\tau$ an involution of the second kind, then $\mathcal{H}_{\tau} \cap \mathcal{H}_{+} \subset G$ and the question of obtaining a unique $\tau$-Iwasawa decomposition reduces to finding a subgroup $\mathcal{H}_{+}$such that this intersection is actually trivial. This is a question for the finite dimensional group $G$, where it is known that one can in some cases find complementary subgroups and in other cases one can not.

4. Kellersch [26] has given an example of a Birkhoff decomposable subgroup and an antilinear involution such the there are two disjoint open orbits. Thus, in general, one cannot assume that $\mathcal{H}_{\tau} \cdot \mathcal{H}_{+}$is open and dense in $\mathcal{H}$. In many examples this will be possible though.

To complete this round of definitions:

Definition 2.9. An element $x \in \mathcal{H}$ is called $\tau$-Iwasawa decomposable if it can be factorized

$$
x=z_{\tau} y_{+},
$$

with $z_{\tau} \in \mathcal{H}_{\tau}$ and $y_{+} \in \mathcal{H}^{+}$. 
Remark 2.10. a) If $\mathcal{H}$ is Iwasawa decomposable, then every element in some open neighbourhood of $I$ is Iwasawa decomposable.

b) A $\tau$-Iwasawa decomposition $x=z_{\tau} y_{+}$as above, can be written as $x y_{+}^{-1}=$ $z_{\tau}=\tau z_{\tau}=\tau x\left(\tau y_{+}\right)^{-1}$, and the factorization (9) is equivalent to the problem of finding $y_{+} \in \mathcal{H}^{+}$such that

$$
x^{-1} \tau x=y_{+}^{-1} \tau y_{+} .
$$

Theorem 2.11. Suppose $\mathcal{H}$ is any Birkhoff decomposable subgroup of an arbitrary loop group $\Lambda G$, and $\tau$ is an involution of the second kind of $\mathcal{H}$. Then

(1) $\mathcal{H}$ is $\tau$-Iwasawa decomposable.

(2) Assume $x^{-1} \tau x \in \mathcal{R B H}$ and Birkhoff decompose $x^{-1} \tau x=v_{+} \cdot a v_{-}$, where $v_{ \pm} \in \mathcal{H}_{1}^{ \pm}$and $a \in \mathcal{H}^{\circ}$. Then $x$ is $\tau$-Iwasawa decomposable if and only if $a=k^{-1} \tau k$ for some $k \in \mathcal{H}^{\circ}$.

(3) If the constant subgroup $\mathcal{H}^{0}$ is connected and compact then every element, $x \in \mathcal{H}$, satisfying the requirement that $x^{-1} \tau x$ is in the identity component of $\mathcal{R B H}$, is $\tau$-Iwasawa decomposable.

(4) The left factor, $z_{\tau}$, in a $\tau$-Iwasawa decomposition (9) of $x \in \mathcal{H}$, is unique up to right multiplication by a constant element of $\mathcal{H}_{\tau}$.

(5) If $x$ is $\tau$-Iwasawa decomposable, then the factors $z_{\tau}$ and $y_{+}$, in the decomposition $x=z_{\tau} y_{+}$, can, in a neighbourhood of $x$, be chosen to depend real analytically on $x$.

Proof. (1) We need to show that $\mathcal{H}_{\tau} \cdot \mathcal{H}^{+}$is open. We apply example 3 of section 2.2. Thus there exists an open (and dense) subset $\Omega$ of $\mathcal{K}=\mathcal{H} \times \mathcal{H}$ such that every element of $\Omega$ can be written uniquely in the form

$$
(h, f)=(u, u) \cdot(a, b) \cdot\left(v_{+}, v_{-}\right) \in \mathcal{K}_{1}^{-} \times \mathcal{K}^{0} \times \mathcal{K}_{1}^{+} .
$$

Now we consider the extension of the given involution, $\tau(f, g):=(\tau g, \tau f)$. Then $\mathcal{K}_{\tau}=\{(h, \tau h), h \in \mathcal{H}\}$, and so we can identify $\mathcal{H}$ with $\mathcal{K}_{\tau}$. If $x=(h, \tau h) \in \mathcal{K}_{\tau}$, then the two components of (11) are $h=u a v_{+}$and $\tau h=u b v_{-}$. Applying $\tau$ to both of these expressions, we also have $\tau h=\tau u \tau a \tau v_{+}$and $h=\tau u \tau b \tau v_{-}$, to give the two decompositions

$$
\begin{aligned}
(h, \tau h) & =(u, u) \cdot(a, b) \cdot\left(v_{+}, v_{-}\right) \\
& =(\tau u, \tau u) \cdot(\tau b, \tau a) \cdot\left(\tau v_{-}, \tau v_{+}\right) \in \mathcal{K}_{1}^{-} \times \mathcal{K}^{0} \times \mathcal{K}_{1}^{+} .
\end{aligned}
$$

Now the uniqueness of the factors in this Birkhoff decomposition implies that $\tau u=$ $u$, and so $\Omega \cap \mathcal{K}_{\tau}$ is just the set $\left\{(h, \tau h)=\left(u a v_{+}, \tau\left(u a v_{+}\right)\right) \mid \tau u=u\right\} \subset \mathcal{K}_{\tau}$. Since $\mathcal{K}_{\tau}$ carries the induced topology, $\Omega \cap \mathcal{K}_{\tau}$ is open in $\mathcal{K}_{\tau}$. Finally, under our identification of $\mathcal{H}$ with $\mathcal{K}_{\tau}$, this open set is nothing but the set $\mathcal{H}_{\tau} \cdot \mathcal{H}^{+}$.

(2) Since $x^{-1} \tau x \in \mathcal{R} \mathcal{B H}$, we can write $x^{-1} \tau x=v_{+} a v_{-}$, where $v_{ \pm} \in \mathcal{H}_{1}^{ \pm}$and $a \in \mathcal{H}^{\circ}$. Now the fact that $\tau\left(x^{-1} \tau x\right)=\left(x^{-1} \tau x\right)^{-1}$ is then equivalent to

$$
v_{-}^{-1} a^{-1} v_{+}^{-1}=\tau v_{+} \tau a \tau v_{-} .
$$

By uniqueness of the factors in the Birkhoff decomposition, one has $v_{-}^{-1}=\tau v_{+}$and $\tau a=a^{-1}$. Hence

$$
x^{-1} \tau x=v_{+} a\left(\tau v_{+}\right)^{-1},
$$

and so the claim follows from Remark 2.10.

(3) Follows, in view of (2), from Proposition 2.12 below. 
(4) Assume we have two decompositions $z y_{+}=h v_{+} \cdot$ Then

$$
h^{-1} z=v_{+} y_{+}^{-1} \text {. }
$$

Clearly, the left element is fixed by $\tau$ and the right one is mapped to $\mathcal{H}^{-}$. Thus $h^{-1} z$ is in the intersection of $\mathcal{H}^{+}$and $\mathcal{H}^{-}$, and is contained in $\mathcal{H}_{\tau} \cap G$. This is the claim.

(5) also follows from Proposition 2.12 since the factors $v_{+}$and $a$ in (12) both depend real analytically on $x$.

To complete the proof of the Theorem above we show:

Proposition 2.12. Let $H$ be a semisimple Lie group and $\tau$ an involution of $H$. Define

$$
\begin{array}{r}
H^{*}:=\left\{x \in H ; \tau x=x^{-1}\right\}, \\
\mathcal{B} H^{*}:=\left\{x \in H^{*} ; \exists k \in H: x=k(\tau k)^{-1}\right\} .
\end{array}
$$

(1) $\mathcal{B H}^{*}$ is open, and, locally, the element $k$ can always be chosen to depend real analytically on $x$.

(2) If $H$ is compact then $\mathcal{B} H^{*}$ is both open and closed.

Proof. Let $H_{\tau}$ denote the fixed point subgroup of $\tau$. Consider the map $f: H / H_{\tau} \rightarrow$ $H^{*}$, given by $f\left(k H_{\tau}\right)=k(\tau k)^{-1}$. To prove both (1) and (2), we show that $f$ is a one to one immersion with open image. Then, for a family $x(t) \in \mathcal{B} H^{*}$, there is a real analytic family $f^{-1}(x(t)) \in H / H_{\tau}$, and, on any contractible set in $H / H_{\tau}$, this lifts to a real analytic family $k(t)$.

Firstly, $a(\tau a)^{-1}=b(\tau b)^{-1} \Leftrightarrow b^{-1} a=\tau\left(b^{-1} a\right) \Leftrightarrow b^{-1} a \in \mathcal{H}_{\tau} \Leftrightarrow a \mathcal{H}_{\tau}=b \mathcal{H}_{\tau}$, which shows that $f$ is one to one.

Secondly, $f$ is an immersion, which can be seen as follows: the tangent space to $H / H_{\tau}$ at $[k]:=k H_{\tau}$ can be written in the form $T_{[k]}\left(H / H_{\tau}\right)=\{r V ; r \in$ $\mathbf{R}, V \in \operatorname{Lie}(H), \tau V=-V\}$, where we think of all groups as matrix groups and $\tau$ is a linear map. To compute the differential of $f$ at $[k]$, it is enough to consider curves of the form $\gamma(t)=[k \exp (t V)]$, where $\tau V=-V$. Then $f(\gamma(t))=$ $k \exp (t V) \exp (t V)(\tau k)^{-1}=k \exp (2 t V)(\tau k)^{-1}=k(\tau k)^{-1}+2 t k V(\tau k)^{-1}+o\left(t^{2}\right)$. Hence $\left.\mathrm{d} f\right|_{[k]}(V)=2 k V(\tau k)^{-1}$. The map $\left[V \mapsto 2 k V(\tau k)^{-1}\right]$ has zero kernel, so $\left.\mathrm{d} f\right|_{[k]}$ is injective.

Thirdly, the spaces have the same dimension, because the tangent space at the identity of $H / H_{\tau}$ is equal to the -1 eigenspace of $\tau$, which is the tangent space at the identity of $H^{*}$. Thus the image of $f$, namely $\mathcal{B} H^{*}$, is open.

Remark 2.13. Analysing the proof of the Theorem above one sees that an involution of the second kind becomes an involution of the first kind, in the double loop group, for which we have already proven some properties. Therefore, in some sense, only involutions of the first kind are of interest to us. The double loop group set up is also appropriate for handling situations where two loops are necessary, rather than one.

\section{CONNECTION ORDER $\left(\begin{array}{l}b \\ a\end{array}\right)$ MAPS AND THEIR SPLitTings}

In this section, we make the same assumptions on $G$ and the topology of $\Lambda G$ as in the previous sections. Let $U$ be a manifold and define $\Lambda G(U)$ to be the set of smooth maps $F: U \rightarrow \Lambda G$. Sometimes one wants to normalize $F$ to assume the 
value $I$ at some preassigned point $t_{0} \in U$. In this case one says that $f$ is based (at $\left.t_{0}\right)$. For any extended integers $a$ and $b$ in $\mathbb{Z} \cup\{ \pm \infty\}$, with $a \leq b$, define $\Lambda G(U)_{a}^{b}$ to be the subset of such maps which are of connection order $\left(\begin{array}{l}b \\ a\end{array}\right)$, that is, whose Maurer-Cartan forms have the Fourier expansion

$$
F^{-1} \mathrm{~d} F=A_{a} \lambda^{a}+\ldots+A_{b} \lambda^{b},
$$

for any $t \in U$. Define $B \Lambda G(U)$ to be the subset of $\Lambda G(U)$ consisting of elements $F$ such that $F(t) \in B \Lambda G$ for any $t$ in $U$, and $B \Lambda G(U)_{a}^{b}$ analogously. Similar notation applies to subgroups and quotients of $\Lambda G$ in the obvious way.

\subsection{Splitting without an involution of the second kind.}

Proposition 3.1. Let $\mathcal{H}$ be any Birkhoff decomposable subgroup of $\Lambda G$, and $a$ and $b$ extended integers with $a<0<b$. To every

$$
F \in B \mathcal{H}(U)_{a}^{b}
$$

there corresponds a pair

$$
G_{-} \in \mathcal{H}(U)_{a}^{-1}, F_{+} \in \mathcal{H}(U)_{1}^{b}
$$

such that

$$
F=F_{+} F_{-}=G_{-} G_{+}
$$

for some $F_{-}$and $G_{+}$in $\mathcal{H}^{-}(U)$ and $\mathcal{H}^{+}(U)$ respectively. If $F$ is assumed to be based at $t_{0}$, then $F_{+}, F_{-}, G_{+}$, and $G_{-}$are also based at $t_{0}$.

Proof. $G_{-}$and $F_{+}$are just the left factors given by the left and right Birkhoff decompositions respectively for $F$.

Let us check that $F_{+} \in \mathcal{H}(U)_{1}^{b}$ : For fixed $t \in U$, the Birkhoff factorization theorem states that the map $\left(F_{+}, F_{-}\right) \mapsto F$ is a diffeomorphism, if we normalize e.g. $F_{+}=I+a_{1} \lambda+\ldots$. So we can deduce that the map $t \mapsto F(t) \mapsto F_{+}(t)$ is also differentiable. Hence $F_{+} \in \mathcal{H}(U)$. Now by definition, $F_{+}$has the Fourier expansion

$$
F_{+}=I+a_{1} \lambda+a_{2} \lambda^{2}+\ldots
$$

valid on $\overline{\mathbf{D}_{+}}$, so

$$
F_{+}^{-1} \mathrm{~d} F_{+}=b_{1} \lambda+b_{2} \lambda^{2}+\ldots
$$

is of bottom degree 1 in $\lambda$. For the top degree, note that $F_{+}$also has the expression

$$
F_{+}=F F_{-}^{-1}
$$

and we are given that

$$
F^{-1} \mathrm{~d} F=A_{a} \lambda^{a}+\ldots+A_{0}+\ldots+A_{b} \lambda^{b} .
$$

Now comparing the Maurer-Cartan forms of both sides of (14),

$$
b_{1} \lambda+b_{2} \lambda^{2}+\ldots=F_{-}\left[A_{a} \lambda^{a}+\ldots+A_{0}+\ldots+A_{b} \lambda^{b}\right] F_{-}^{-1}+F_{-} \mathrm{d}\left(F_{-}^{-1}\right),
$$

and using the fact that both $F_{-}$and $F_{-}^{-1}$ are in $\Lambda^{-} G$, the conclusion is that both sides are of top degree $b$ in $\lambda$.

The proof that $G_{-} \in \mathcal{H}(U)_{a}^{-1}$ is similar.

Finally, assume that $F$ is based at $t_{0} \in U$. Then $I=F\left(t_{0}, \lambda\right)=F_{+}\left(t_{0}, \lambda\right) G_{-}\left(t_{0}, \lambda\right)$ for all $\lambda$. Hence $F_{+}\left(t_{0}, \lambda\right)=F_{0}$ and $G_{-}\left(t_{0}, \lambda\right)=G_{0}$ are constant loops, and inverse to each other. By the expression (13) for $F_{+}$, it follows that $F_{0}=G_{0}=I$.

The following result is a little stronger than a converse to the last proposition: 
Proposition 3.2. Let $\mathcal{H}$ be any Birkhoff decomposable subgroup. Suppose $a$ and $b$ are extended integers with $a<0<b$. To every pair

$$
G_{-} \in \mathcal{H}(U)_{a}^{\infty}, F_{+} \in \mathcal{H}(U)_{-\infty}^{b},
$$

such that $F_{+}^{-1} G_{-} \in B \mathcal{H}(U)$, there corresponds a unique element

$$
F \in B \mathcal{H}(U)_{a}^{b}
$$

which satisfies the equations

$$
\begin{aligned}
F & =F_{+} F_{-} \\
& =G_{-} G_{+},
\end{aligned}
$$

for some $F_{-}$and $G_{+}$in $\mathcal{H}^{-}(U)$ and $\mathcal{H}^{+}(U)$ respectively. Moreover, if $F_{+}$and $G_{-}$ are based at $t_{0}$, then $F$ is also based at $t_{0}$.

Proof. By assumption, for each $t \in U$ there is a unique Birkhoff factorization

$$
F_{+}^{-1} G_{-}=F_{-} G_{+}^{-1}
$$

where $F_{-}$and $G_{+}$are maps from $U$ to $\mathcal{H}^{-}$and $\mathcal{H}^{+}$respectively and $F_{-}$has constant term $I$. Rearranging this, we see that $F$ is well-defined by the equations (15) and (16).

Now calculate the Maurer-Cartan form for $F$, using (15):

$$
F^{-1} \mathrm{~d} F=F_{-}^{-1}\left(F_{+}^{-1} \mathrm{~d} F_{+}\right) F_{-}+F_{-}^{-1} \mathrm{~d} F_{-} .
$$

Because $F_{+} \in \mathcal{H}(U)_{-\infty}^{b}$ and $F_{-}$has only powers of $1 / \lambda$, this expression has top degree at most $b$ in $\lambda$, and, using (16), we similarly deduce that $F^{-1} \mathrm{~d} F$ also has bottom degree $a$ in $\lambda$. In other words, $F \in \mathcal{H}(U)_{a}^{b}$. Assume now that $F_{+}$and $G_{-}$ are based at $t=t_{0}$, then we have

$$
F\left(t_{0}, \lambda\right)=F_{-}\left(t_{0}, \lambda\right)=G_{+}\left(t_{0}, \lambda\right),
$$

and this is a constant function of $\lambda$ by holomorphicity. Since $F_{-}(t, \infty)=I$, this constant is the identity, and so $F$ is also based at $t_{0}$.

3.2. Splitting with an involution of the second kind. If $\mathcal{H}$ is Birkhoff decomposable, then, clearly, Proposition 3.1 applies to the case $F \in B \mathcal{H}_{\tau}(U)_{a}^{b}$, where $\tau$ is an involution of the second kind, although $G_{-}$and $F_{+}$will not be fixed by $\tau$ themselves. In fact, if $\tau F=F$, we have

$$
\tau F_{+} \tau F_{-}=G_{-} G_{+} .
$$

Since $\tau$ interchanges \pm , this presents two representations of an element in $\mathcal{H}^{-} \cdot \mathcal{H}^{+}$. If the constant terms in the expansions of both $F_{+}$and $G_{-}$are taken to be the identity, uniqueness of the left Birkhoff factorization implies that $G_{-}=\tau F_{+}$.

With this in mind, we can state the analogue of Proposition 3.2 for a loop group with an involution, $\tau$, of the second kind. This depends on the $\tau$-Iwasawa factorization result, Theorem 2.11 and for this reason we define the Iwasawa big cells,

$$
\mathcal{B}_{+}^{\tau} \mathcal{H}=\mathcal{H}_{\tau} \cdot \mathcal{H}^{+}, \quad \mathcal{B}_{-}^{\tau}=\mathcal{H}_{\tau} \cdot \mathcal{H}^{-}
$$

and $\mathcal{B}_{ \pm}^{\tau} \mathcal{H}(U)_{a}^{b}$ to be the set of connection order $\left(\begin{array}{l}b \\ a\end{array}\right)$ maps from $U$ which take values in $\mathcal{B}_{ \pm}^{\tau} \mathcal{H}$. This is the exact analogue to the Birkhoff case, except that in general the Iwasawa cells are not dense. For many cases, they have been investigated in [26]. Further, if $\tau$ is an anti-linear involution defining a compact real form, then one can 
use the standard Iwasawa decomposition to obtain the result without restrictions on $F$ globally, as in [16.

Remark 3.3. The map $x(\lambda) \mapsto x\left(\lambda^{-1}\right)$ is an analytic isomorphism of $\Lambda G$. It follows that all the earlier definitions and results about Iwasawa decompositions are also valid if we switch + and - and $\lambda=0$ with $\lambda=\infty$.

Proposition 3.4. Let $\mathcal{H}$ be any Birkhoff decomposable subgroup of $\Lambda G$, and $\tau$ an involution of the second kind of $\mathcal{H}$. Suppose $b$ is an extended integer greater than zero. Given an element

$$
F_{+} \in \mathcal{B}_{-}^{\tau} \mathcal{H}(U)_{-\infty}^{b}
$$

there exists a unique element

$$
[F] \in \frac{\mathcal{H}_{\tau}}{\mathcal{H}_{\tau}^{\circ}}(U)_{-b}^{b}
$$

such that for any local frame for $[F]$, that is, an element $F \in \mathcal{H}_{\tau}(W)_{-b}^{b}$, which represents $[F](t)$ for all $t$ in $W \subset U$, one has the decomposition

$$
\begin{aligned}
F & =F_{+} F_{-} \\
& =\tau F_{+} \tau F_{-},
\end{aligned}
$$

for some $F_{-}$in $\mathcal{H}^{-}(W)$. Moreover, if $F_{+}$is based at $t_{0}$, then $F$ and $F_{-}$can be chosen to be based at $t_{0}$ also.

Proof. By the definition of the Iwasawa big cell $\mathcal{B}_{-}^{\tau} \mathcal{H}$, one has, for each $t \in U$ the Iwasawa decomposition

$$
F_{+}=F F_{-}^{-1}
$$

where $F(t) \in \mathcal{H}_{\tau}$. By Theorem 2.11 (combined with Remark 3.3), $F(t)$ is unique up to right multiplication by $\mathcal{H}_{\tau}^{\circ}$, which makes $[F](t)$ unique. By Item $(5)$ of the same theorem, $F$ can locally be chosen as a smooth function of $t$, and therefore the equivalence class $[F] \in \mathcal{H}_{\tau} / \mathcal{H}_{\tau}^{\circ}(U)$ is smooth. Since $F$ is fixed by $\tau$, we have $F=F_{+} F_{-}=\tau F_{+} \tau F_{-}$. To check that $F$ is of connection order $\left({ }_{-b}^{b}\right)$, note that it follows from the expression $F=F_{+} F_{-}$, where $F_{+}^{-1} \mathrm{~d} F_{+}$is of top degree $b$ and $F_{-}$ takes values in $\mathcal{H}^{-}$, that $F^{-1} \mathrm{~d} F=\ldots+\alpha_{b} \lambda^{b}$ is of top degree $b$. Then $F^{-1} \mathrm{~d} F=$ $\tau\left(F^{-1} \mathrm{~d} F\right)=\beta_{-b} \lambda^{-b}+\ldots$ is of bottom degree $-b$, due to the assumption that $\tau$ is an involution of the second kind. Finally, if $F_{+}\left(t_{0}\right)=I$, then $I=I \cdot I$ is a valid Iwasawa decomposition, so one can choose $F\left(t_{0}\right)=F_{-}\left(t_{0}\right)=I$.

3.3. Splitting with more than one involution of the second kind. If we need to deal with the fixed point set of several (mutually commuting) involutions of the second kind, then we can always reduce this to the case of one involution of the second kind, together with one or more additional involutions of the first kind. It is straightforward to check the following facts:

Lemma 3.5. Let $\mathcal{H}$ be any subgroup of $\Lambda G$, and suppose $\tau$ and $\hat{\tau}$ are a pair of commuting involutions of the second kind of $\mathcal{H}$. Define an involution

$$
\rho:=\tau \circ \hat{\tau} \text {. }
$$

Then

(1) $\rho$ is an involution of the first kind.

(2) If $\hat{\tau}$ is complex anti-linear and $\tau$ is complex linear then $\rho$ is complex antilinear. 
(3) $\mathcal{H}_{\tau \hat{\tau}}=\mathcal{H}_{\rho \hat{\tau}}=\mathcal{H}_{\rho \tau}$.

\section{Potentials}

In many applications of geometric interest one wants to use the method presented in this paper to construct examples with specific properties. In the context of the previous section one could say that one tries to find appropriate pairs of maps $F_{+}$ and $G_{-}$as in Proposition 3.2 or one map $F_{+}$as in Proposition 3.4. The results just quoted then produce an "extended frame $F$ ", from which the geometric object is derived (more or less directly: see, e.g.[16] and section 6 and 7). While, in general, the basic maps mentioned above are simpler than the frame $F$ constructed from them, it is not trivial at all to write down those basic maps either. But one can simplify the situation by one more step: one can consider the infinitesimal versions of the basic maps.

Definition 4.1. Let $\mathcal{H}$ be any Birkhoff decomposable subgroup of $\Lambda G$, and $a$ and $b$ extended integers with $a<0<b$. Let $F \in B \mathcal{H}(U)_{a}^{b}$ and $G_{-} \in \mathcal{H}(U)_{a}^{-1}$ and $F_{+} \in \mathcal{H}(U)_{1}^{b}$, such that $F=F_{+} F_{-}=G_{-} G_{+}$, and $\left.F_{+}\right|_{\lambda=0}=\left.G_{-}\right|_{\lambda=\infty}=I$. Then the pair $\left(G_{-}, F_{+}\right)$will be called a basic pair for $F$, and the pair of Maurer-Cartan forms

$$
\eta=\left(G_{-}^{-1} d G_{-}, F_{+}^{-1} d F_{+}\right)=\left(\eta_{-}, \eta_{+}\right)
$$

will be called a potential or a pair of potentials for $F$. Similarly, for $\mathcal{H}_{\tau}$, where $\tau$ is an involution of the second kind, and $\eta_{-}=\tau\left(\eta_{+}\right)$, as in Proposition 3.4. we call $F_{+}$a basic map, and its Maurer-Cartan form $\eta_{+}$a potential.

Remark 4.2. (1) Of course, $\eta$ satisfies the obvious integrability condition. In some cases, such as 16, the integrability for $\eta$ is trivial, while the integrability condition for $F^{-1} d F$ is highly non-trivial.

(2) In most cases one starts from some frame $F$ which is smooth on the manifold $U$. The Birkhoff splitting used to derive the basic maps is not global and thus frequently introduces singularities. In some cases, such as in [16, one can modify the construction of the basic pairs so that one obtains smooth basic pairs. In general it is not known, whether for a smooth $F$ one can always find modified smooth basic pairs.

(3) One should consider $\eta$ as a global object on $U$ which has singularities.

(4) What, in the case of harmonic maps, has been called the DPW method is as follows: start conversely, from some potential $\eta$ which has the proper expansion relative to $\lambda$ and satisfies the required integrability condition. Next one will integrate the equations $\mathrm{d} G_{-}=G_{-} \eta_{-}$and $\mathrm{d} F_{+}=F_{+} \eta_{+}$, with some initial condition. If $\eta$ is free of singularities and if $U$ is simply connected, these differential equations have global solutions. The corresponding frame constructed in Section 3, will, in general, still have singularities, but, wherever it is smooth, it will have the right properties. At any rate, starting from some potentials with singularities one obtains geometric frames with singularities and vice versa.

(5) In some geometric cases it is necessary that singularities occur, such as the case of constant negative Gauss curvature surfaces in Euclidean 3-space, where Hilbert proved that there are no complete immersions. For the case of $\mathcal{H}_{\tau}$, when the underlying Lie group is not compact, so that the Iwasawa decomposition is not global, one can start with a potential which is smooth 
everywhere, and such that the basic map $F_{+}$is everywhere regular, but the extended frame $F$ has singularities at points where the Iwasawa decomposition breaks down. This is investigated for CMC surfaces in Minkowski space in 9 .

(6) Clearly, the question of whether singularities can be removed or appear necessarily is a major problem that needs to be discussed.

(7) Sometimes creating frames from potentials is confused with dressing (see the corresponding section below). We would therefore like to point out that knowing all potentials is (on a theoretical level) equivalent with knowing all frames. Dressing only creates new surfaces from old ones. It is a group action on the space of frames. In all known cases, the dressing action has many different orbits, and one usually does not know representatives for all these orbits. On the other hand finding potentials which reflect, to some extent, the properties one is looking for and dressing the corresponding frames has frequently lead to good results.

\section{Dressing SOlutions}

One of the methods for producing new solutions of connection order $\left(\begin{array}{l}b \\ a\end{array}\right)$ maps from given solutions has been the dressing action of Zakharov and Shabat [38. It was first discussed in terms of harmonic maps into Lie groups by Uhlenbeck [37. See 21] and 32] for more details and references. In the case of Harmonic maps, a more general dressing action by subgroups of $\Lambda \mathrm{GL}(n, \mathbb{C})$ is treated in [4].

5.1. Dressing for connection order $\left(\begin{array}{l}b \\ 1\end{array}\right)$ maps. If $\mathcal{H}$ is Birkhoff decomposable, there is a natural local action of $\mathcal{H}^{-}$on $\mathcal{H}(U)_{1}^{b}$, where $U$ is some manifold, (and analogously of $\mathcal{H}^{+}$on $\mathcal{H}(U)_{a}^{-1}$ ) defined as follows: if $F_{+} \in \mathcal{H}(U)_{1}^{b}$ and $g_{-} \in \mathcal{H}^{-}$, then set $U^{*}=\left\{t \in U \mid g F_{+}(t) \in \mathcal{R B \mathcal { H }}\right\}$. Then, for each $t \in U^{*}$, define a new element $\hat{F}_{+}(t)$ in $\mathcal{H}_{1}^{+}$by the Birkhoff factorization

$$
g_{-}(\lambda) F_{+}(t, \lambda)=\hat{F}_{+}(t, \lambda) h_{-}(t, \lambda),
$$

Applying Proposition 3.1 to the map $F=g_{-} F_{+}$, which is clearly of type $\left({ }_{-\infty}^{b}\right)$, it follows that $\hat{F}_{+}$is an element of $\mathcal{H}(U)_{1}^{b}$. It is straightforward to check that this defines a left action by $\mathcal{H}^{-}$on $\mathcal{H}(U)_{1}^{b}$, or more precisely a local action as one has to replace $U$ with $U^{*}$, which is, in general, some large open subset of $U$. Moreover, if $F_{+}$is based at $t_{0}$, then $\hat{F}_{+}$is also based at $t_{0}$.

5.2. Dressing for general order $\left(\begin{array}{l}b \\ a\end{array}\right)$ maps. The above dressing action extends in a natural way to a left action of $\mathcal{H}^{-} \times \mathcal{H}^{+}$on $\mathcal{H}(U)_{a}^{b}$, where $a<0<b$. If $\left(g_{-}, g_{+}\right) \in \mathcal{H}^{-} \times \mathcal{H}^{+}$and $F \in \mathcal{H}(U)_{a}^{b}$, then $\hat{F}:=\left(g_{-}, g_{+}\right) \diamond(F)$ is obtained on some subset $U^{*}$ of $U$ by first solving the pair of equations

$$
\begin{aligned}
& g_{-} F=\hat{F}_{+} h_{-}, \\
& g_{+} F=\hat{G}_{-} h_{+},
\end{aligned}
$$

where the right hand sides are right and left Birkhoff factorizations respectively. If $F$ is of order $\left(\begin{array}{l}b \\ a\end{array}\right)$ then $\hat{F}_{+}$and $\hat{G}_{-}$are of order $\left(\begin{array}{l}b \\ 1\end{array}\right)$ and $\left(\begin{array}{c}-1 \\ a\end{array}\right)$ respectively, so, applying Proposition 3.2 we can find a new element $\hat{F} \in(U)_{a}^{b}$ which satisfies

$$
\begin{aligned}
\hat{F} & =\hat{F}_{+} \hat{F}_{-} \\
& =\hat{G}_{-} \hat{G}_{+},
\end{aligned}
$$


for some $\hat{F}_{-}$and $\hat{G}_{+}$.

Bearing in mind the principle that $F$ is associated to a pair $F_{+} \in \mathcal{H}(U)_{1}^{b}$ and $G_{-} \in \mathcal{H}(U)_{a}^{-1}$ via Propositions 3.1 and 3.2 , it is unsurprising that this is the same action which one obtains by applying the action described above of $g_{-}$to $F_{+}$and $g_{+}$to $G_{-}$separately, as the reader may easily verify.

The action can also be rephrased simply by using Example 3 of Section 2.2 what was described above simply is the dressing action of $\mathcal{K}^{+}$on $(F, F) \in \mathcal{K}_{1}^{-}$:

$$
\left(h^{+}, h^{-}\right)(F, F)=(\hat{F}, \hat{F})\left(v_{+}, v_{-}\right) .
$$

\section{ISOMETRIC IMMERSIONS OF SPACE FORMS INTO A SPHERE}

Here we introduce the loop group formulation for the problem of isometric immersions of space forms into a sphere. The formulation for immersions into hyperbolic space is analogous, replacing the group $S O(n+k+1)$ with the Lorentz group $S O_{-1}(n+k+1)$, and we describe that in Section 7.1 below. In this section we are primarily summarizing results from [19] and [8; the key result is Proposition 6.16,

6.1. Extended frames and connections. As pointed out just above, we are interested in all immersions $f: M_{c} \rightarrow \mathbb{S}^{n}$, where $M_{c}$ denotes some space form of curvature $c$ and $\mathbb{S}^{N}$ denotes the unit sphere in $R^{N+1}$. Wherever we say "immersion", the map is assumed to be of class $\mathcal{C}^{\infty}$. Since such immersions do not exist in all cases globally, but on large submanifolds which one obtains by removing "singular points of $f$ " from $M_{c}$, we consider submanifolds $U \subset M_{c}$, where one can think of $\mathrm{U}$ as being almost all of $M_{c}$. For convenience we can even assume $U$ to be simply connected without changing the point of view above. In the discussion of adapted frames below, one considers maps into a homogeneous space for which a global frame need not necessarily exist. If one is interested in global problems, then this can be handled in the present setting by considering maps into the the homogeneous space $\mathcal{H} / \mathcal{H}^{\circ}$, as in [6]. For convenience, we instead assume that $U$ is a contractible open subset of $\mathbf{R}^{n}$, with basepoint $t_{0}$.

Let now $f: U \rightarrow \mathbb{S}^{n+k}$ be an immersion. Then we will say $F: U \rightarrow S O(n+k+1)$ is an adapted frame for $f$ if the $(n+1)^{\prime}$ 'th column of $F$ is $f$ and if the derivative $\mathrm{d} f$ has no component in the directions given by the last $k+1$ columns. This means that if $f$ is an immersion and

$$
F=\left[e_{1}, \ldots, e_{n}, \xi_{1}, \ldots, \xi_{k+1}\right],
$$

(where $\xi_{1}=f$ ), then $\left\{e_{i}\right\}$ and $\left\{\xi_{i}\right\}$ are orthonormal bases for the tangent and normal spaces respectively of $f(U) \subset \mathbb{R}^{n+k+1}$. Note that an adapted frame always exists on a contractible set $U$.

In general, there is no canonical choice of adapted frame for a given map $f$. The freedom is characterized as follows: consider the order two automorphisms of $S O(n+k+1, \mathbb{C})$ given by

$$
\begin{gathered}
\sigma_{2}=\operatorname{Ad}_{P}, \\
\tau_{2}=\operatorname{Ad}_{Q},
\end{gathered}
$$

for the matrices

$$
P:=\left[\begin{array}{cc}
I_{n \times n} & 0 \\
0 & -I_{(k+1) \times(k+1)}
\end{array}\right], Q:=\left[\begin{array}{cc}
I_{(n+1) \times(n+1)} & 0 \\
0 & -I_{k \times k}
\end{array}\right] .
$$


Here $I_{r}$ denotes an $r \times r$ identity matrix. Let $S O(n+k+1, \mathbb{R})_{\sigma \tau}$ denote the elements of $S O(n+k+1, \mathbb{R})$ fixed by both $\sigma_{2}$ and $\tau_{2}$.

Lemma 6.1. If $F: U \rightarrow S O(n+k+1, \mathbb{R})$ is an adapted frame for $f: U \rightarrow \mathbb{S}^{n+k}$, then any other adapted frame for $f$ is given by

$$
\hat{F}=F T,
$$

where $T: U \rightarrow S O(n+k+1, \mathbb{R})_{\sigma \tau}$.

Proof. A change of oriented orthonormal bases for the tangent and normal frames, keeping the $(n+1)$ 'th column (which is the map $f$ ) fixed, amounts to right multiplication by a matrix of the form

$$
T=\left[\begin{array}{ccc}
T_{1} & 0 & 0 \\
0 & 1 & 0 \\
0 & 0 & T_{2}
\end{array}\right],
$$

with $T_{1} \in S O(n, \mathbb{R})$ and $T_{2} \in S O(k, \mathbb{R})$. But these are precisely the elements of $S O(n+k+1, \mathbb{R})$ which are fixed by both $\sigma_{2}$ and $\tau_{2}$.

Let $\Omega^{1}(U)$ be the space of real-valued one-forms on $U$ and denote by $A=$ $F^{-1} \mathrm{~d} F \in \operatorname{so}(n+k+1) \otimes \Omega^{1}(U)$ the pull-back by $F$ of the left Maurer-Cartan form of $S O(n+k+1)$. More explicitly,

$$
A=\left[\begin{array}{cc}
\omega & \hat{\beta} \\
-\hat{\beta}^{T} & \eta
\end{array}\right]
$$

where $\omega \in \operatorname{so}(n) \otimes \Omega^{1}(U)$ and $\eta \in s o(k+1) \otimes \Omega^{1}(U)$. If $f$ is an immersion, then $\omega, \eta$ and $\hat{\beta}$ are the Levi-Civita connection form, the normal connection form and the second fundamental form respectively of the immersion $f$, considered as a submanifold of $\mathbb{R}^{n+k}$. The requirement that $\mathrm{d} f$ has no component in the direction of any of the last $k+1$ columns is equivalent to the statement that the first row and first column of $\eta$ consist of zeros.

If $\mathfrak{g}$ is the Lie algebra of a Lie group $G$, and $A$ is any 1-form in $\mathfrak{g} \otimes \Omega^{1}(U)$ on the simply connected manifold $U$, then the condition for the (global) existence of a map $F$ into $G$ with connection 1-form $A$ is the Maurer-Cartan equation

$$
\mathrm{d} A+A \wedge A=0 .
$$

Thus adapted frames are in one-to-one correspondence with 1-forms $A \in \operatorname{so}(n+k+$ 1) $\otimes \Omega^{1}(U)$ which satisfy the Maurer-Cartan equation and have the property that the first row and column of $\eta$ in (25) are zero. We will call such one-forms adapted connection forms.

If we write

$$
\hat{\beta}=\left[\begin{array}{ll}
\theta & \beta
\end{array}\right],
$$

where $\theta$ is the column vector $\left[\theta_{i}:=e_{i}^{T} \mathrm{~d} f\right]$ and $\beta$ is the second fundamental form of the map into $\mathbb{S}^{n+k}$, then $f$ being immersive is equivalent with the 1 -forms $\theta_{i}$ being linearly independent. In a neighbourhood of such a point, the induced sectional curvatures on the immersed submanifold are of constant value $c$ if and only if

$$
\mathrm{d} \omega+\omega \wedge \omega=c \theta \wedge \theta^{T} .
$$


Remark 6.2. 1. For the case $c<1$, the smallest possible codimension for even the local existence of such an immersion is $k=n-1$, and in this case it is known [29] that the normal bundle is flat:

$$
\mathrm{d} \eta+\eta \wedge \eta=0 .
$$

Therefore, in the rest of this paper, we will impose the condition (27) for all cases, as it comes naturally with the integrable system described below and thus allows us to apply integrable systems methods.

2. Since we are going to discuss methods to produce adapted frames (without necessarily satisfying the immersion condition), we define a slight generalization of an isometric immersion with flat normal bundle of a space form into $\mathbb{S}^{n+k}$; generically, and in the appropriate codimension, these will be isometric immersions.

Definition 6.3. A constant curvature $c$ map with flat normal bundle into $\mathbb{S}^{n+k}$ is a map $f: U \rightarrow \mathbb{S}^{n+k}$ corresponding to an adapted frame as described above, and where the equations (26) and (27) hold.

It was shown, in [19] for the case $c \neq 0$ and [8] for the case $c=0$, that one can insert an (appropriate complex or real) auxiliary parameter $\lambda$ into the 1-form $A$, such that the assumption that the Maurer-Cartan equation holds for all $\lambda$ is equivalent not only to the global existence of $F$ on the simply connected manifold $U$ mentioned above, but also to the equations (26) and (27), where $c$ will depend on $\lambda$ in general. The relevant facts are outlined below.

\subsection{Extended frames for flat immersions.}

Lemma 6.4. Suppose that

$$
A^{\lambda}=\left[\begin{array}{cc}
\omega & \lambda \hat{\beta} \\
-\lambda \hat{\beta}^{T} & \eta
\end{array}\right]
$$

is a family of complex matrix-valued one-forms, where $\omega, \eta$ and $\hat{\beta}$ are independent of $\lambda$. Then $A^{\lambda}$ satisfies the Maurer-Cartan equation for all $\lambda$ if and only if

(1) $A^{\lambda_{0}}$ satisfies the Maurer-Cartan equation for some $\lambda_{0} \in \mathbf{C}^{*}$,

(2) $\mathrm{d} \omega+\omega \wedge \omega=0$ and

(3) $\mathrm{d} \eta+\eta \wedge \eta=0$

Remark 6.5. Lemma 6.4 is verified by writing out the Maurer-Cartan equation for $A^{\lambda}$ and comparing coefficients of like powers of $\lambda$, and the same comment applies to Lemma 6.8 below.

Definition 6.6. A type $A$ extended connection on $U$ is a family of $s o(n+k+1, \mathbb{R})$ valued 1 -forms $A^{\lambda}$ of the form (28), where

(1) $A^{\lambda}$ is real valued for real values of $\lambda$,

(2) $\omega$ is $n \times n$,

(3) $\omega=0$ and $\eta=0$,

(4) $A^{\lambda}$ satisfies the Maurer-Cartan equation for all $\lambda$.

Note that a type A extended connection yields a family of adapted connection forms parameterized by $\lambda \in \mathbf{R}^{*}$. These integrate to give adapted frames whose $(n+1)^{\prime}$ th columns are constant curvature 0 maps with flat normal bundle, or flat maps. Assuming the initial condition

$$
F\left(t_{0}, \lambda\right)=I
$$


at some base point $t_{0} \in U$, the family of adapted frames $F(\lambda, t)$ corresponding to the extended connection $A^{\lambda}$ is unique.

Conversely, given a flat map $f$ into $\mathbb{S}^{n+k}$, one can choose an adapted frame $F$ for $f$ and obtains the Maurer Cartan form $A=F^{-1} d F$, which is of the form

$$
A=\left[\begin{array}{cc}
\omega & \hat{\beta} \\
-\hat{\beta}^{T} & \eta
\end{array}\right]
$$

Introducing $\lambda$ as in (28) above verifies that this connection form is integrable for all $\lambda$.

On the other hand, it is known that for a given flat immersion $f$ into $\mathbb{S}^{n+k}$, one can choose an adapted frame $F$ for $f$ such that both the tangent and the normal bundles are parallel, so that $\omega=0$ and $\eta=0$. Given an initial condition $F\left(t_{0}\right)=I$, this parallel frame is unique. Multiplying the corresponding connection form by a parameter $\lambda$ gives a unique type A extended connection.

It is worth noting here that the transition from any adapted frame for a flat map to one with parallel bundles can be done independently of $\lambda$, and this argument applies to any connection order $\left(\begin{array}{l}b \\ 0\end{array}\right)$ map, gauging it to an order $\left(\begin{array}{l}b \\ 1\end{array}\right)$ map:

Lemma 6.7. If $F(\lambda, t)$ is a family of adapted frames whose Maurer-Cartan forms satisfy all the conditions of Definition 6.6 with the exception of (3), then then there is a unique map $G: U \rightarrow S O(n+k+1)$ with $G\left(t_{0}\right)=I$ such that $F(\lambda, t) G(t)$ is parallel, that is, its Maurer-Cartan form satisfies all the conditions of Definition 6.6 .

Proof. If $A=A_{0}+A_{1} \lambda$ then the Maurer-Cartan equation for $A$ is

$$
\mathrm{d} A_{0}+A_{0} \wedge A_{0}+\left(\mathrm{d} A_{1}+A_{0} \wedge A_{1}+A_{1} \wedge A_{0}\right) \lambda+A_{1} \wedge A_{1} \lambda^{2}=0 .
$$

Since this holds for all $\lambda$, the 1 -form $A_{0}$ itself satisfies the Maurer-Cartan equation and there exists a map $H: U \rightarrow S O(n+k+1)$ such that

$$
A_{0}=H^{-1} \mathrm{~d} H \text {. }
$$

Now set $G=H^{-1}$ and we have

$$
\begin{aligned}
(F G)^{-1} \mathrm{~d}(F G) & =H\left(F^{-1} \mathrm{~d} F\right) H^{-1}+H \mathrm{~d}\left(H^{-1}\right) \\
& =H A_{1} H^{-1} \lambda .
\end{aligned}
$$

Since $A_{0}$ is of the form $\left[\begin{array}{cc}\omega & 0 \\ 0 & \eta\end{array}\right], H$ has the form $\left[\begin{array}{cc}G_{1} & 0 \\ 0 & G_{2}\end{array}\right]$, and therefore

$$
(F G)^{-1} \mathrm{~d}(F G)=\left[\begin{array}{cc}
0 & \lambda G_{1} \hat{\beta} G_{2}^{T} \\
-\lambda G_{2} \hat{\beta}^{T} G_{1}^{T} & 0
\end{array}\right]
$$

which is a type A extended connection.

6.3. Extended frames for immersions of non-flat space forms. Here we summarize results from [19.

Lemma 6.8. Suppose that

$$
\left.B^{\lambda}=\left[\begin{array}{ccc}
-\left[\left(\lambda+\lambda^{-1}\right) \theta\right. & \left(\lambda-\lambda^{-1}\right) \beta
\end{array}\right]^{T} \begin{array}{cc}
\left(\lambda+\lambda^{-1}\right) \theta & \left.\left(\lambda-\lambda^{-1}\right) \beta\right]
\end{array}\right]
$$

is a family of complex matrix-valued one-forms, where $\omega, \theta, \eta$ and $\beta$ are independent of $\lambda$. Then $B^{\lambda}$ satisfies the Maurer-Cartan equation for all $\lambda$ if and only if 
(1) $B^{\lambda_{0}}$ satisfies the Maurer-Cartan equation for some $\lambda_{0} \in \mathbf{C}^{*}$,

(2) $\mathrm{d} \omega+\omega \wedge \omega=4 \theta \wedge \theta^{T}$.

(3) $\mathrm{d} \eta+\eta \wedge \eta=0$.

Remark 6.9. In the case of isometric immersions, $\theta$ is an $n \times 1$ column matrix and the coframe for the immersion $f$ will be

$$
\hat{\theta}=\left(\lambda+\lambda^{-1}\right) \theta
$$

so we will need that $\lambda \neq \pm i$ (for an immersion) and the condition at item 2 of the lemma means constant sectional curvature

$$
c^{\lambda}=\frac{4}{\left(\lambda+\lambda^{-1}\right)^{2}} .
$$

Definition 6.10. A type $B_{j}$ extended connection on $\mathbb{R}^{n}$ is a family of $s o(n+k+$ $1, \mathbb{C}$ )-valued 1 -forms $B^{\lambda}$ of the form (30), where

(1) $\omega$ is $n \times n$,

(2) $\theta$ is $n \times 1$,

(3) the first row and column of $\eta$ are zero,

(4) $B^{\lambda}$ satisfies the Maurer-Cartan equation for all $\lambda$ and

(5) the matrix coefficients of $B^{\lambda}$ satisfy the reality condition $\mathbf{R}_{j}$, chosen from the following three possibilities:

$$
\begin{array}{cccc}
\mathbf{R}_{1}: & \left(\rho_{1} X\right)(\lambda):=\overline{X(-\bar{\lambda})}=X(\lambda) & \Longleftrightarrow & X(\lambda) \text { real for } \lambda \in i \mathbf{R}^{*}, \\
\mathbf{R}_{2}: \quad\left(\rho_{2} X\right)(\lambda):=\overline{X(\bar{\lambda})}=X(\lambda) & \Longleftrightarrow \quad X(\lambda) \text { real for } \lambda \in \mathbf{R}^{*}, \\
\mathbf{R}_{-1}: \quad\left(\rho_{-1} X\right)(\lambda):=\overline{X(1 / \bar{\lambda})}=X(\lambda) & \Longleftrightarrow \quad X(\lambda) \text { real for } \lambda \in \mathbb{S}^{1},
\end{array}
$$

Remark 6.11. As in the flat case, type $B_{1}, B_{2}$ and $B_{-1}$ extended connections correspond to families of adapted connection forms parameterised this time by $\lambda$ in $i \mathbf{R}^{*}, \mathbf{R}^{*}$ and $\mathbb{S}^{1}$ respectively. Lemma 6.8 implies that the corresponding maps $f^{\lambda}$ are constant curvature $c^{\lambda}$ maps, with flat normal bundle, where $c^{\lambda}=\frac{4}{\left(\lambda+\lambda^{-1}\right)^{2}}$. Further, one has the following ranges for $c^{\lambda}$ :

$$
\begin{aligned}
& \lambda \in i \mathbf{R}^{*} \Rightarrow c^{\lambda} \in(-\infty, 0), \\
& \lambda \in \mathbf{R}^{*} \Rightarrow c^{\lambda} \in(0,1], \\
& \lambda \in \mathbb{S}^{1} \Rightarrow c^{\lambda} \in(1, \infty) .
\end{aligned}
$$

Definition 6.12. Type $A$ and type $B_{j}$ extended frames are the families of adapted frames obtained from the type A and type $B_{j}$ extended connections defined above, by integrating them with the initial condition $F\left(t_{0}, \lambda\right)=I$ for each $\lambda$.

Also as in the flat case, any constant curvature $c$ map, for $c \neq 0$, corresponds to a type $B_{1}, B_{2}$ or $B_{-1}$ extended frame depending on the value of $c$. Unlike the flat case, where we could choose a parallel frame, here, even with the normalization $F\left(t_{0}, \lambda\right)=I$, the extended frame is only determined up to a right multiplication by a matrix in $S O(n+k+1, \mathbb{R})_{\sigma \tau}$.

6.4. Type $\mathbf{A}$ extended frames with the reality condition $\mathbf{R}_{1}$. We defined extended frames of type A as certain families of maps into the Lie group with reality condition $\mathbf{R}_{2}$. However we could also have used the first reality condition as follows: if $A^{\lambda}=A_{0}+A_{1} \lambda$, with $A_{i}$ real and $\mathrm{d} A+A \wedge A=0$, define

$$
\hat{A}^{\lambda}:=A_{0}+i A_{1} \lambda \text {. }
$$


Then $\hat{A}$ also satisfies the Maurer-Cartan equation and, for $\lambda=r_{0} \in \mathbb{R}$, one has

$$
A^{r_{0}}=\hat{A}^{-i r_{0}} \text {. }
$$

So $\hat{A}^{\lambda}$ integrates to a family of adapted frames of flat maps for $\lambda \in i \mathbf{R}^{*}$. We therefore define extended frames of type $A 1$ and A2 accordingly. Note that extended frames of type A with reality condition $\mathbf{R}_{3}$ do not exist.

Let $\left\{e_{k}\right\}$ be the standard basis for $\mathbb{R}^{n+k+1}$. If $I$ is one of the intervals

$$
I_{1}=(-\infty, 0), \quad I_{2}=(0,1), \quad I_{-1}=(1, \infty),
$$

let $\mathbb{S}_{I}\left(U, t_{0}\right)$ be the set of families of constant curvature $c \in I$ maps with flat normal bundle $f: U \rightarrow \mathbb{S}^{n+k}$ such that $f\left(t_{0}\right)=e_{n+1}$, and $\mathbb{S}_{0}\left(U, t_{0}\right)$ the analogous set for flat maps.

We summarize the preceding discussion as:

Proposition 6.13. $\quad(1)$ The elements of $\mathbb{S}_{0}\left(U, t_{0}\right)$ are in one to one correspondence with type $A_{j}$ extended frames, where $j$ is 1 or 2.

(2) The elements of $\mathbb{S}_{I_{j}}\left(U, t_{0}\right)$ are in one to one correspondence with equivalence classes of type $B_{j}$ extended frames, where $j$ is 1, 2, or -1 . The equivalence is up to right multiplication by a map $T: U \rightarrow S O(n+k+1, \mathbb{R})_{\sigma \tau}$.

Remark 6.14. The statement in the second part of the proposition above is frequently rephrased as "The elements of $\mathbb{S}_{I_{j}}\left(U, t_{0}\right)$ are in one to one correspondence with the gauge orbits of the $\lambda$-independent gauge group on the space of frames."

6.5. Loop group formulation. The above extended connections can be defined fairly naturally as 1-forms with values in subspaces of certain loop algebras as follows: if $\mathfrak{g}$ is any Lie algebra, let $\widetilde{\mathfrak{g}}:=\mathfrak{g}\left[\lambda, \lambda^{-1}\right]$, the Lie algebra of Laurent polynomials with coefficients in $\mathfrak{g}$, which is given the Lie bracket

$$
\left[\sum_{i=\alpha}^{\beta} X_{i} \lambda^{i}, \sum_{i=\gamma}^{\delta} Y_{i} \lambda^{i}\right]:=\sum_{i, j}\left[X_{i}, Y_{j}\right] \lambda^{i+j} .
$$

If $\widetilde{\mathfrak{H}}$ is any algebra of Laurent series in $\lambda$, then let us define $\widetilde{\mathfrak{H}}_{a}^{b}$ to be the vector subspace consisting of elements whose lowest power of $\lambda$ is $a$ and highest power of $\lambda$ is $b$.

Suppose we are given commuting Lie algebra automorphisms $\sigma_{k}$ and $\tau_{2}$ of $\mathfrak{g}$ of order $k$ and 2 respectively, where $k \geq 2$. Using these, define commuting automorphisms $\sigma$ and $\tau$ of the same order on $\widetilde{\mathfrak{g}}$ as follows:

$$
\begin{array}{r}
(\sigma X)(\lambda):=\sigma_{k}\left(X\left(\left(\zeta_{k}\right)^{-1} \lambda\right)\right), \\
(\tau X)(\lambda):=\tau_{2}(X(1 / \lambda)),
\end{array}
$$

where $\zeta_{k}$ is a primitive $k$ 'th root of unity. Let $\widetilde{\mathfrak{g}}_{\sigma}$ be the subalgebra of $\widetilde{\mathfrak{g}}$ consisting of elements fixed by $\sigma$, and define $\tilde{\mathfrak{g}}_{\sigma \tau}$ to be the subalgebra whose elements are fixed by both automorphisms. Assuming $\sigma$ and $\tau$ also commute with the involutions $\rho_{i}$, we further define $\widetilde{\mathfrak{g}}_{\sigma} \mathbf{R}_{j}$ and $\widetilde{\mathfrak{g}}_{\sigma \tau} \mathbf{R}_{j}$ to be the corresponding real subalgebras with the reality condition $\mathbf{R}_{j}$.

Now consider the case $\mathfrak{g}=s o(n+k+1, \mathbb{C})$ and the inner automorphisms $\sigma_{2}=$ $\operatorname{Ad}_{P}$ and $\tau_{2}=\operatorname{Ad}_{Q}$ defined earlier by (22) and (23).

It is straightforward to verify the following: 
Lemma 6.15. (1) For $j=1,2$, type $A_{j}$ extended connections are precisely the one-forms which satisfy the Maurer-Cartan equation and whose coefficients are in $\left(\widetilde{\mathfrak{g}}_{\sigma} \mathbf{R}_{j}\right)_{1}^{1}$.

(2) For $j=1,2,3$ type $B_{j}$ extended connections are precisely the one-forms which satisfy the Maurer-Cartan equation and whose coefficients are in $\left(\widetilde{\mathfrak{g}}_{\sigma \tau} \mathbf{R}_{j}\right)_{-1}^{1}$.

Suppose $\sigma_{k}$ and $\tau_{2}$ are commuting automorphisms of $G$, of order $k$ and 2 respectively, and denote the corresponding Lie algebra automorphisms with the same notation. Extend $\sigma_{k}$ and $\tau_{2}$ to automorphisms $\sigma$ and $\tau$ of $\Lambda G$ by (32) and (33), and define $\Lambda G_{\sigma}$ and $\Lambda G_{\sigma \tau}$ to be the subgroups of $\Lambda G$ consisting of elements which are fixed by $\sigma$, and by both $\sigma$ and $\tau$ respectively. Similarly we have the real subgroups $\Lambda G_{\sigma} \mathbf{R}$ and $\Lambda G_{\sigma \tau} \mathbf{R}$ where $\mathbb{R}$ is one of the reality conditions defined previously, with Lie algebras $\Lambda \mathfrak{g}_{\sigma} \mathbf{R}$ and $\Lambda \mathfrak{g}_{\sigma \tau} \mathbf{R}$ respectively. Thus Lemma6.15]and Proposition6.13 can be rephrased as:

Proposition 6.16. (1) For $j=1,2$, the elements of $\mathbb{S}_{0}\left(U, t_{0}\right)$ are in one to one correspondence with the elements of $\Lambda G_{\sigma} \mathbf{R}_{j}(U)_{1}^{1}$ that are based at $t_{0}$.

(2) Let $\mathcal{H}=\Lambda G_{\sigma \tau} \mathbf{R}_{j}$. For $j=1,2$ and -1 , the elements of $\mathbb{S}_{I_{j}}\left(U, t_{0}\right)$ are in one to one correspondence with the elements of $\frac{\mathcal{H}}{\mathcal{H}^{\circ}}(U)_{-1}^{1}$ that are based at $t_{0}$.

\section{Correspondence Between Constant positive, Negative And Zero CURVATURE MAPS INTO A SPHERE OR HYPERBOLIC SPACE}

We now show how the above loop group splitting results can be used to reduce the local problem of constant curvature immersions with flat normal bundle into $\mathbb{S}^{m}$ and $\mathbb{H}^{m}$ to the flat case.

7.1. Constant curvature immersions into hyperbolic space. It turns out that the splitting results for connection order $\left(\begin{array}{l}b \\ a\end{array}\right)$ maps applied to immersions of space forms into the sphere lead naturally to immersions into hyperbolic space, in the case of the reality condition of the second kind, $\mathbf{R}_{-1}$. We need the following discussion to explain this. Define the Lie group $S O_{-1}(n+k+1, \mathbb{C})$ to be the subgroup of $G L(n+k+1, \mathbb{C})$ consisting of matrices satisfying the equation

$$
\begin{array}{r}
X^{T} J X=J, \\
J:=\operatorname{diag}\left(I_{n \times n},-1, I_{k \times k}\right) .
\end{array}
$$

Extended frames for constant curvature isometric immersions into hyperbolic space $\mathbb{H}^{n+k}$ are defined analogously to those for the sphere in Section 6.2, replacing the Lie group $S O(n+k+1)$ with $S O_{-1}(n+k+1)$. See [19] for a discussion of this in the non-flat case. Define $\mathbb{H}_{0}\left(U, t_{0}\right)$ and $\mathbb{H}_{I}\left(U, t_{0}\right)$ analogously to their $\mathbb{S}$ counterparts, replacing the sphere with hyperbolic space. The analogues of the results in Section 6 hold, replacing $\mathbb{S}$ with $\mathbb{H}$ and $G=S O(n+k+1, \mathbb{C})$ with $H=S O_{-1}(n+k+1, \mathbb{C})$; in addition, the formula for the induced curvature $c_{\lambda}$ is changed by a minus sign, so that one must also replace $I_{j}$ with its reflection $-I_{j}$, for statements involving curvature in the interval $I_{j}$. Thus the analogue to Proposition 6.16 is:

Proposition 7.1. (1) For $j=1,2$, the elements of $\mathbb{H}_{0}\left(U, t_{0}\right)$ are in one to one correspondence with the elements of $\Lambda H_{\sigma} \mathbf{R}_{j}(U)_{1}^{1}$ that are based at $t_{0}$. 
(2) Let $\mathcal{H}=\Lambda H_{\sigma \tau} \mathbf{R}_{j}$. For $j=1,2$, or -1 , the elements of $\mathbb{H}_{-I_{j}}\left(U, t_{0}\right)$ are in one to one correspondence with the elements of $\frac{\mathcal{H}}{\mathcal{H}^{\circ}}(U)_{-1}^{1}$ that are based at $t_{0}$.

Moreover, maps into $\Lambda G_{\sigma}$ with a particular reality condition can be interpreted as maps into $\Lambda H_{\sigma}$ with a different reality condition via the next proposition. In order to state the result more symmetrically, we introduce one further reality condition of the second kind:

$\mathbf{R}_{-2}:\left(\rho_{-2} X\right)(\lambda):=\overline{X(-1 / \bar{\lambda})}=X(\lambda)$.

Proposition 7.2. Consider the involutions of the first kind, defined by:

$$
\begin{aligned}
\left(\hat{\rho}_{1} X\right)(\lambda) & :=\tau \circ \rho_{-1} X(\lambda) \\
& =Q \overline{X(\bar{\lambda})} Q^{-1} . \\
\left(\hat{\rho}_{2} X\right)(\lambda) & :=\tau \circ \rho_{-2} X(\lambda) \\
& =Q \overline{X(-\bar{\lambda})} Q^{-1},
\end{aligned}
$$

Define $\phi: G L(n+k+1, \mathbb{C}) \rightarrow G L(n+k+1, \mathbb{C})$ by

$$
\begin{array}{r}
\phi X:=\operatorname{Ad}_{T} X, \\
T:=\operatorname{diag}\left(i I_{n \times n}, 1, i I_{k \times k}\right) .
\end{array}
$$

Then

(1) $\phi$ is an isomorphism between the subgroups $G:=S O(n+k+1, \mathbb{C})$ and $H:=S O_{-1}(n+k+1, \mathbb{C})$.

(2) $\phi$ defines a bijection

$$
\Lambda G_{\sigma \hat{\rho}_{j}}(U)_{1}^{1} \leftrightarrow \Lambda H_{\sigma} \mathbf{R}_{j}(U)_{1}^{1},
$$

for $j=1,2$.

(3) $\phi$ defines a bijection

$$
\Lambda G_{\sigma \tau} \mathbf{R}_{j}(U)_{-1}^{1} \leftrightarrow \Lambda H_{\sigma \tau} \mathbf{R}_{-j}(U)_{-1}^{1},
$$

for $j=1,2$.

Proof. Let $\hat{F}:=\phi F$.

(1) Since $\operatorname{Ad}_{T}$ is a homomorphism, and clearly bijective, it is enough to verify that $F^{T} F=I$ is equivalent to $\hat{F}^{T} J \hat{F}=J$ which is straightforward.

(2) Let $F$ be an element of $\Lambda G_{\sigma \hat{\rho}_{j}}(U)_{1}^{1}$. To show that $\hat{F}$ is an element of $\Lambda H_{\sigma} \mathbf{R}_{j}(U)_{1}^{1}$, we need to show

(a) $\hat{F}$ is of connection order $\left(\begin{array}{l}1 \\ 1\end{array}\right)$,

(b) $\left(\rho_{j} \hat{F}\right)(\lambda)=\hat{F}(\lambda)$, and

(c) $(\sigma \hat{F})(\lambda)=P \hat{F}(-\lambda) P^{-1}=\hat{F}(\lambda)$.

All of these follow in a straightforward manner from the corresponding properties of $F$. We verify the reality conditions here using the fact that $F$ is fixed by both $\tau$ and $\hat{\rho}_{j}$, that is:

$$
\begin{array}{r}
F(\lambda)=P F(-\lambda) P, \\
F(\lambda)=\overline{Q F\left((-1)^{j+1} \bar{\lambda}\right)} Q,
\end{array}
$$


as well as the readily verified identities

$$
\begin{array}{r}
P Q T=-\bar{T} \\
\quad=-T^{-1} .
\end{array}
$$

Thus we have

$$
\begin{aligned}
\left(\rho_{j} \hat{F}\right)(\lambda) & :=\overline{\hat{F}\left((-1)^{j} \bar{\lambda}\right)} \\
& =T^{-1} \overline{F\left((-1)^{j} \bar{\lambda}\right)} T \\
& =T^{-1} Q F(-\lambda) Q T \\
& =T^{-1} Q P F(\lambda) P Q T \\
& =(-T) F(\lambda)\left(-T^{-1}\right) \\
& =\hat{F}(\lambda)
\end{aligned}
$$

(3) This is also straightforward, the main work being to check that if $F \in$ $\Lambda G_{\sigma \tau} \mathbf{R}_{j}$ then $\hat{F}:=T F T^{-1}$ is fixed by both $\sigma$ and $\rho_{-j}$.

Item (3) of Proposition 7.2 has the following immediate corollary:

Proposition 7.3. There are natural identifications

$$
\begin{aligned}
\mathbb{S}_{(-\infty, 0)}\left(U, t_{0}\right) & \longleftrightarrow \mathbb{H}_{(-\infty,-1)}\left(U, t_{0}\right) \\
\mathbb{S}_{(1, \infty)}\left(U, t_{0}\right) & \longleftrightarrow \mathbb{H}_{(0, \infty)}\left(U, t_{0}\right)
\end{aligned}
$$

7.2. Applying the splitting results to isometric immersions. We state the results locally, although similar statements would hold globally, away from some singular set.

Definition 7.4. Let $k \geq n-1$. The set of germs of constant curvature zero maps into $\mathbb{S}^{n+k}$, at $t_{0} \in U$ is defined by

$$
\mathbb{S}_{0}\left(t_{0}\right):=\left(\bigcup_{U} \mathbb{S}_{0}\left(U, t_{0}\right)\right) / \sim,
$$

where the union is over all neighbourhoods $U$ of $t_{0}$, and two maps are equivalent if they agree on some neighbourhood of $t_{0}$.

For $j=1,2$ or -1 , let $\mathbb{S}_{I_{j}}\left(t_{0}\right)$ be the analogous set of germs corresponding to families of constant curvature $c$ maps, with $c \in I_{j}$.

Theorem 7.5. (1) There are canonical bijections

$$
\mathbb{S}_{(-\infty, 0)}\left(t_{0}\right) \longleftrightarrow \mathbb{S}_{0}\left(t_{0}\right) \longleftrightarrow \mathbb{S}_{(0,1)}\left(t_{0}\right)
$$

(2) There is a canonical bijection

$$
\mathbb{S}_{(1, \infty)}\left(t_{0}\right) \longleftrightarrow \mathbb{H}_{0}\left(t_{0}\right)
$$

Proof. (1) An element of $\mathbb{S}_{(-\infty, 0)}\left(t_{0}\right)$ or $\mathbb{S}_{(0,1)}\left(t_{0}\right)$ corresponds by Proposition 6.16 to an element $[F] \in \frac{\Lambda G_{\sigma \tau} \mathbf{R}}{\Lambda G_{\sigma \tau} \mathbf{R}^{0}}(W)_{-1}^{1}$, based at $t_{0}$, and where $W$ is some neighbourhood of $t_{0}$, and $\mathbf{R}$ is $\mathbf{R}_{1}$ or $\mathbf{R}_{2}$ respectively. By Proposition 3.1 this corresponds to a unique element $F_{+} \in \Lambda G_{\sigma} \mathbf{R}(W)_{1}^{1}$, also based at $t_{0}$, and which, according to Proposition 6.16 gives an element of $\mathbb{S}_{0}\left(t_{0}\right)$.

Conversely, given a based element $F_{+} \in \Lambda G_{\sigma} \mathbf{R}(W)_{1}^{1}$, using Proposition 6.16 again and Proposition 3.4 there is a unique element of $\frac{\Lambda G_{\sigma \tau} \mathbf{R}}{\Lambda G_{\sigma \tau} \mathbf{R}^{0}}(W)_{-1}^{1}$, based at $t_{0}$. 
(2) For the case $c \in(1, \infty)$, recall that constant curvature $c$ maps are represented by maps into the loop group with the reality condition $\mathbf{R}_{-1}$, which is of the second kind and commutes with $\tau$.

Using Lemma 3.5, the analogue of part (1) of this theorem gives local correspondences between these constant curvature maps and based elements of $\Lambda G_{\sigma \hat{\rho}_{1}}(U)_{1}^{1}$, where $\hat{\rho}_{1}$ is the anti-linear involution of the first kind given by 35 .

Using the bijection at item (2) of Proposition 7.2 ,

$$
\Lambda G_{\sigma \hat{\rho}_{1}}(U)_{1}^{1} \leftrightarrow \Lambda H_{\sigma} \mathbf{R}_{1}(U)_{1}^{1},
$$

we obtain the required correspondence.

Finally, the analogue of Theorem 7.5 for immersions into hyperbolic space, whose proof is essentially the same is:

Theorem 7.6. (1) There are canonical bijections

$$
\mathbb{H}_{(0, \infty)}\left(t_{0}\right) \longleftrightarrow \mathbb{H}_{0}\left(t_{0}\right) \longleftrightarrow \mathbb{H}_{(-1,0)}\left(t_{0}\right)
$$

(2) There is a canonical bijection

$$
\mathbb{H}_{(-\infty,-1)}\left(t_{0}\right) \longleftrightarrow \mathbb{S}_{0}\left(t_{0}\right)
$$

7.3. Example. It is difficult to exhibit examples with explicit formulae: given an isometric immersion, there is a recipe for inserting the parameter $\lambda$ into the MaurerCartan form of an adapted frame, but then one can generally only integrate this Maurer-Cartan form numerically. Further, the Birkhoff and Iwasawa splittings must generally be computed numerically. In the case of CMC surfaces, software has been written which can produce images of the surface corresponding to any "potential" used in the DPW method.

Here we consider one simple example, from [6], of a family of 2-dimensional spheres, of constant curvature $c_{\lambda} \in(1, \infty)$, isometrically embedded in $\mathbb{S}^{3}$, and we compute the corresponding flat immersion. Consider the family of maps $F_{\lambda}: \mathbf{R}^{2} \rightarrow$ $G=S O(4, \mathbf{C})$ which takes $(u, v) \in \mathbf{R}^{2}$ to the matrix

$$
\left[\begin{array}{cccc}
\cos u & -\sin u \sin v & a \sin u \cos v & b \sin u \cos v \\
0 & \cos v & a \sin v & b \sin v \\
-a \sin u & -a \cos u \sin v & a^{2} \cos u \cos v+b^{2} & a b(\cos u \cos v-1) \\
-b \sin u & -b \cos u \sin v & a b(\cos u \cos v-1) & b^{2} \cos u \cos v+a^{2}
\end{array}\right]
$$

where

$$
a=\frac{1}{2}\left(\lambda+\lambda^{-1}\right), \quad b=\frac{i}{2}\left(\lambda-\lambda^{-1}\right) .
$$

The Maurer-Cartan form of $F_{\lambda}$ is

$$
F_{\lambda}^{-1} \mathrm{~d} F_{\lambda}=\left[\begin{array}{cccc}
0 & -\sin v \mathrm{~d} u & a \cos v \mathrm{~d} u & b \cos v \mathrm{~d} u \\
\sin v \mathrm{~d} u & 0 & a \mathrm{~d} v & b \mathrm{~d} v \\
-a \cos v \mathrm{~d} u & -a \mathrm{~d} v & 0 & 0 \\
-b \cos v \mathrm{~d} u & -b \mathrm{~d} v & 0 & 0
\end{array}\right] .
$$

It is easy to check that $F_{\lambda}^{-1} \mathrm{~d} F_{\lambda}$ is fixed by $\sigma, \tau$ and $\rho_{-1}$, so it takes values in the Lie algebra of $\mathcal{H}:=\Lambda G_{\sigma \tau} \mathbf{R}_{-1}$. Because $F_{\lambda}(0,0)=I \in \mathcal{H}$, it follows that $F_{\lambda}$ is a map into $\mathcal{H}$, and, since its Maurer-Cartan form has top and bottom degree 1 and -1 respectively, it represents an element of $\frac{\mathcal{H}}{\mathcal{H}^{\circ}}\left(\mathbf{R}^{2}\right)_{-1}^{1}$. Thus, according to Section 
6. if the the third column $f^{\lambda}$ of $F_{\lambda}$ is an immersion, then, for a value of $\lambda$ in $\mathbb{S}^{1}$, it is an immersion into $\mathbb{S}^{3}$ with constant Gauss curvature greater or equal to 1 . The coframe for $f^{\lambda}$ is given by

$$
\theta=\left[\begin{array}{c}
\frac{1}{2}\left(\lambda+\lambda^{-1}\right) \cos v \mathrm{~d} u \\
\frac{1}{2}\left(\lambda+\lambda^{-1}\right) \mathrm{d} v
\end{array}\right]
$$

and so, if $\lambda \neq \pm i$, then $f^{\lambda}$ is immersive away from the degenerate coordinate lines $\cos v=0$. In fact $f^{\lambda}$ is a deformation, through a family of isometrically embedded spheres, of the totally geodesic embedding of $\mathbb{S}^{2}$ into $\mathbb{S}^{3}$ given by $f(u, v)=$ $\left[\begin{array}{lll}\sin u \cos v, & \sin v, \quad \cos u \cos v, \quad 0\end{array}\right]^{T}$, which is achieved at $\lambda=1$.

To understand the corresponding flat immersion $f_{+}$into $\mathbb{H}^{3}$, given by the second part of Theorem [7.5, let

$$
F=F_{+} F_{-}
$$

represent the right Birkhoff splitting of the frame $F$, valid on some open set $U \subset \mathbf{R}^{2}$. Then $F_{+}$is an adapted frame for $f_{+}$. Expressing $F_{+}^{-1} \mathrm{~d} F_{+}$in terms of $F^{-1} \mathrm{~d} F$ and the function $F_{-}$, we deduce that

$$
F_{+}^{-1} \mathrm{~d} F_{+}=K \alpha_{+} K^{-1} \lambda
$$

where $F^{-1} \mathrm{~d} F=\alpha_{-} \lambda^{-1}+\alpha_{0}+\alpha_{+} \lambda$ and $K$ is a map into $\mathcal{H}^{\circ}=S O(2) \times S O(2)$, represented by diagonal matrices whose components are $2 \times 2$ blocks.

Thus we have

$$
\begin{array}{r}
\alpha_{+}=\left[\begin{array}{cc}
0 & A \\
-A^{T} & 0
\end{array}\right], \quad A=\frac{1}{2}\left[\begin{array}{ccc}
\cos v \mathrm{~d} u & i \cos v \mathrm{~d} u \\
\mathrm{~d} v & i \mathrm{~d} v
\end{array}\right], \\
K=\left[\begin{array}{cccc}
\cos \theta & \sin \theta & 0 & 0 \\
-\sin \theta & \cos \theta & 0 & 0 \\
0 & 0 & \cos \phi & \sin \phi \\
0 & 0 & -\sin \phi & \cos \phi
\end{array}\right],
\end{array}
$$

where $\theta$ and $\phi$ are functions $U \rightarrow \mathbf{R}$. Putting these together, we have

$$
\begin{array}{r}
F_{+}^{-1} \mathrm{~d} F_{+}=\left[\begin{array}{cc}
0 & B \\
-B^{T} & 0
\end{array}\right] \lambda=: \eta_{+} \lambda, \\
B=\frac{1}{2}\left[\begin{array}{cc}
e^{i \phi}(\cos \theta \cos v \mathrm{~d} u+\sin \theta \mathrm{d} v) & i e^{i \phi}(\cos \theta \cos v \mathrm{~d} u+\sin \theta \mathrm{d} v) \\
e^{i \phi}(-\sin \theta \cos v \mathrm{~d} u+\cos \theta \mathrm{d} v) & i e^{i \phi}(-\sin \theta \cos v \mathrm{~d} u+\cos \theta \mathrm{d} v)
\end{array}\right] .
\end{array}
$$

Now $F_{+}^{-1} \mathrm{~d} F_{+}$must satisfy the Maurer-Cartan equation for all values of $\lambda$, which is equivalent, in this case, to the pair of equations $\eta_{+} \wedge \eta_{+}=0$ and $\mathrm{d} \eta_{+}=0$. In particular, all the components of the matrix $B$ can be integrated, and we can write

$$
B=\left[\begin{array}{cc}
\mathrm{d} x & i \mathrm{~d} x \\
\mathrm{~d} y & i \mathrm{~d} y
\end{array}\right],
$$

for a pair of functions $x$ and $y$ which map $U \rightarrow \mathbf{R}$ (we take $U$ to be simply connected). It follows that, for a fixed value of $\lambda$ in $i \mathbf{R}, F_{+}$is a part of the torus in $S O(4, \mathbb{C})$ given by exponentiating the Abelian subalgebra, $\mathfrak{m}$ of $s o(4, \mathbb{C})$, given by the span over $\mathbf{R}$ of the two matrices

$$
X=\left[\begin{array}{cccc}
0 & 0 & 1 & i \\
0 & 0 & 0 & 0 \\
-1 & 0 & 0 & 0 \\
-i & 0 & 0 & 0
\end{array}\right], \quad Y=\left[\begin{array}{cccc}
0 & 0 & 0 & 0 \\
0 & 0 & 1 & i \\
0 & -1 & 0 & 0 \\
0 & -i & 0 & 0
\end{array}\right]
$$


The surface in $\mathbb{H}^{3}$ which corresponds to the third column of $F_{+}$, is the analogue in $\mathbb{H}^{3}$ of the Clifford torus in $\mathbb{S}^{3}$. Explicitly, $\left.F_{+}=\exp (x X \lambda) \exp (y Y \lambda)\right)$, and the third column of this matrix is $\left[x \lambda, y \lambda, \frac{1}{2}\left(2-x^{2} \lambda^{2}-y^{2} \lambda^{2}\right),-\frac{1}{2} i\left(x^{2} \lambda^{2}+y^{2} \lambda^{2}\right)\right]^{T}$. After applying $\operatorname{Ad}_{T}$, as given in the proof of Proposition 7.2 this becomes:

$$
f_{+}=\left[i x \lambda, \quad i y \lambda, \frac{1}{2}\left(2-x^{2} \lambda^{2}-y^{2} \lambda^{2}\right), \frac{1}{2}\left(x^{2} \lambda^{2}+y^{2} \lambda^{2}\right)\right]^{T} .
$$

which indeed takes values in $\mathbb{H}^{3}$ for $\lambda \in i \mathbf{R}$.

Remark 7.7. In the application of the DPW method to harmonic maps from a Riemann surface to a symmetric space [16], the basic map (corresponding to $F_{+}$ here) is a holomorphic map into the complex loop group $\Lambda G$. The same construction works for pluriharmonic maps [15]. Note that in our application to space forms, $F_{+}$maps into some real form of $\Lambda G$, and is of course not holomorphic. However, as shown in [6], after complexifying the source manifold, $F_{+}$can easily be extended to a holomorphic map into $\Lambda G$, provided $F_{+}$is real analytic. Taking this as the basic map in the reverse direction of the DPW method, one then obtains an extended frame for a pluriharmonic map. For the example exhibited here, this pluriharmonic map would be from some open subset in $\mathbf{C}^{2}$ into the symmetric space $\frac{S O(4)}{S O(2) \times S O(2)}$.

\section{REFERENCES}

1. M J Ablowitz, D J Kaup, A C Newell, and H Segur, The inverse scattering transform Fourier analysis for nonlinear problems, Stud. Appl. Math. 53 (1974), 249-315.

2. V Balan and J Dorfmeister, Birkhoff decompositions and Iwasawa decompositions for loop groups, Tohoku Math. J. 53 (2001), 593-615.

3. J Bausch and G Rousseau, Algèbres de Kac-Moody affines: Involutions de première espèce des algèbres affines, Inst. Èlie Cartan 11 (1989), 125-139.

4. M J Bergvelt and M A Guest, Actions of loop groups on harmonic maps, Trans. Amer. Math. Soc. 326 (1991), 861-886.

5. N Bourbaki, Groupes et algèbres de Lie, Ch. 1-9, 1960-1982.

6. D Brander, Curved flats, pluriharmonic maps and constant curvature immersions into pseudo-Riemannian space forms, Ann. Global Anal. Geom. 32 (2007), 253-275, DOI 10.1007/s10455-007-9063-y.

7. _ Grassmann geometries in infinite dimensional homogeneous spaces and an application to reflective submanifolds, Int. Math. Res. Not. (2007), rnm092-38, DOI: 10.1093/imrn/rnm092.

8. $\ldots$-symmetric AKS systems and flat immersions into spheres, J. London Math. Soc. (2008), doi: 10.1112/jlms/jdm109.

9. D Brander, N Schmitt, and W Rossman, Holomorphic representation of constant mean curvature surfaces in Minkowski space, Preprint.

10. F E Burstall, Harmonic tori in spheres and complex projective spaces, J. Reine Angew. Math. 469 (1995), 149-177.

11. F E Burstall, D Ferus, F Pedit, and U Pinkall, Harmonic tori in symmetric spaces and commuting Hamlitonian systems on loop algebras, Ann. of Math. (2) 138 (1993), 173-212.

12. F E Burstall, U Hertrich-Jeromin, F Pedit, and U Pinkall, Curved flats and isothermic surfaces, Math. Z. 225 (1997), 199-209.

13. J Dorfmeister, Generalized Weierstraß representations of surfaces, To appear in: Surveys on Geometry and Integrable Systems, Advanced Studies in Pure Mathematics.

14. J Dorfmeister and U Eitner, Weierstrass-type representation of affine spheres, Abh. Math. Sem. Univ. Hamburg 71 (2001), 225-250.

15. J Dorfmeister and J H Eschenburg, Pluriharmonic maps, loop groups and twistor theory, Ann. Global Anal. Geom. 24 (2003), 301-321.

16. J Dorfmeister, F Pedit, and $\mathrm{H} \mathrm{Wu}$, Weierstrass type representation of harmonic maps into symmetric spaces, Comm. Anal. Geom. 6 (1998), 633-668. 
17. J Dorfmeister and $\mathrm{H} \mathrm{Wu}$, Constant mean curvature surfaces and loop groups, J. Reine Angew. Math. 440 (1993), 43-76.

18. D Ferus and F Pedit, Curved flats in symmetric spaces, Manuscripta Math. 91 (1996), 445454.

19. 329-342.

20. D Ferus, F Pedit, U Pinkall, and I Sterling, Minimal tori in $S^{4}$, J. Reine Angew. Math. 429 (1992), 1-47.

21. M A Guest, Harmonic maps, loop groups, and integrable systems, London Mathematical Society Student Texts, vol. 38, Cambridge University Press, 1997.

22. E Heintze, Real forms and finite order automorphisms of affine Kac-Moody algebras - an outline of a new approach, (2007), arXiv:math/0712.2320.

23. F Hélein and $\mathrm{P}$ Romon, Hamiltonian stationary Lagrangian surfaces in $\mathbf{C}^{2}$, Comm. Anal. Geom. 10 (2002), 79-126.

24. N J Hitchin, Harmonic maps from $T^{2}$ to $S^{3}$, Adv. Ser. Math. Phys. 4 (1986), 103-112.

25. V Kac and D Peterson, On geometric invariant theory for infinite-dimensional groups, Lecture Notes in Math. 1271 (1987), 109-142.

26. P Kellersch, Eine Verallgemeinerung der Iwasawa Zerlegung in Loop Gruppen, Differential Geometry-Dynamical Systems. Monographs. Geometry Balkan Press, Bucharest 4 (2004).

27. I M Krichever, An analogue of the d'Alembert formula for the equations of a principal chiral field and the sine-Gordon equation, Dokl. Akad. Nauk SSSR 253 (1980), no. 2, 288-292.

28. F Levstein, A classification of involutive automorphisms of an affine Kac-Moody Lie algebra, J. Algebra 114 (1988), no. 2, 489-518.

29. J D Moore, Isometric immersions of space forms in space forms, Pacific J. Math. 40 (1972), 157-166.

30. U Pinkall and I Sterling, On the classification of constant mean curvature tori, Ann. of Math. (2) 130 (1989), 407-451.

31. A Pressley and G Segal, Loop groups, Oxford Math. Monographs, Clarendon Press, Oxford, 1986.

32. C L Terng, Geometries and symmetries of soliton equations and integrable elliptic equations, arXiv:math/0212372 (2002).

33. C L Terng and K Uhlenbeck, The $n \times n$ KDV flows, (2006), arXiv:nlin/0611006.

34. M Toda, Pseudospherical surfaces via moving frames and loop groups, $\mathrm{PhD}$ Thesis, University of Kansas, 2000.

35. —_ Weierstrass-type representation of weakly regular pseudospherical surfaces in Euclidean space, Balkan J. Geom. Appl. 7 (2002), 87-136.

36. Initial value problems of the sine-Gordon equation and geometric solutions, Ann. Global Anal. Geom. 27 (2005), no. 3, 257-271.

37. K Uhlenbeck, Harmonic maps into Lie groups: classical solutions of the chiral model, J. Differential Geom. 30 (1989), 1-50.

38. V E Zakharov and A B Shabat, Integration of non-linear equations of mathematical physics by the inverse scattering method, II, Funct. Anal. Appl. 13 (1979), 166-173.

Department of Mathematics, Faculty of Science, Kobe University, 1-1, Rokkodai, NADA-KU, KOBE 657-8501, JAPAN

E-mail address: brander@math.kobe-u.ac.jp

Tu Muenchen, Zentrum Mathematik (M8), Boltzmannstr. 3, 85748, Garching, GerMANY

E-mail address: dorfm@ma.tum.de 